FTPI-MINN-14/1, UMN-TH-3321/14

January 26

\title{
Two-Dimensional Sigma Models Related to Non-Abelian Strings in Super-Yang-Mills]
}

Dedicated to the $100^{\text {th }}$ birthday of Isaak Yakovlevich Pomeranchuk

\author{
M. Shifman ${ }^{a}$ and A. Yung ${ }^{a, b}$ \\ ${ }^{a}$ William I. Fine Theoretical Physics Institute, University of Minnesota, \\ Minneapolis, MN 55455, USA \\ ${ }^{b}$ Petersburg Nuclear Physics Institute, Gatchina, St. Petersburg 188300, \\ Russia
}

\begin{abstract}
We review diverse two-dimensional models emerging on the world sheet of non-Abelian strings in the low-energy limit. Non-Abelian strings are supported in a class of four-dimensional bulk theories with or without supersymmetry. In supersymmetric bulk theories we are mostly interested in BPS-saturated strings. Some of these two-dimensional models, in particular, heterotic models, were scarcely studied in the past, if at all. Our main emphasis is on the heterotic $\mathcal{N}=(0,2)$ models. We describe their large- $N$ solution. We briefly comment on $\mathcal{N}=(0,1)$ models although so far they are not obtained on the the world sheet of non-Abelian strings.
\end{abstract}

\footnotetext{
${ }^{1}$ Invited paper, to be published in the Pomeranchuk Memorial Volume (2014).
} 


\section{Contents}

1 Introduction 4

2 How world-sheet models appear:

the simplest example

3 Supersymmetry in the bulk and BPS strings 9

4 Basic models

4.1 Geometric formulation . . . . . . . . . . . . . . . . . . 10

4.2 Gauged formulation ............................. 12

$4.3 \mathrm{CP}(1)$ : a special case .................. 14

5 Witten's large- $N$ solution 15

5.1 Non-supersymmetric $\mathrm{CP}(\boldsymbol{N}-\mathbf{1}) \ldots \ldots \ldots \ldots$

$5.2 \quad$ Supersymmetric $\mathrm{CP}(\boldsymbol{N}-\mathbf{1}) \ldots \ldots \ldots \ldots$

6 Twisted masses 19

7 Large- $N$ solutions with twisted masses 24

$7.1 \mathrm{CP}(\boldsymbol{N}-1)$ with $Z_{N}$ symmetric masses . . . . . . . . . . 24

7.2 Supersymmetric $\mathrm{CP}(\boldsymbol{N}-\mathbf{1})$ with $\mathcal{N}=(\mathbf{2}, \mathbf{2}) \ldots \ldots 27$

7.3 Curves of marginal stability in (2.2) $\mathrm{CP}(\boldsymbol{N}-\mathbf{1})$ with $\boldsymbol{Z}_{\boldsymbol{N}}$ twisted masses .......................... 29

8 Weighted $\operatorname{CP}(N, M)$ models and $z n$ model

9 Heterotic models 35

9.1 How heterotic models appear . . . . . . . . . . . . 36

9.2 Minimal vs. nonminimal $\operatorname{CP}(\boldsymbol{N}-\mathbf{1})$ models with $\mathcal{N}=(\mathbf{0}, \mathbf{2})$

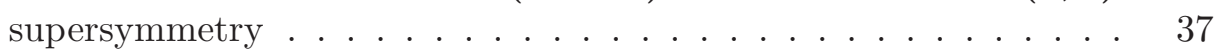

9.2.1 Geometric formulation . . . . . . . . . . . . . 37

9.2 .2 Gauged formulation . . . . . . . . . . . . . 39

9.2.3 Twisted masses . . . . . . . . . . . . . . . . 40

9.3 Beta functions . . . . . . . . . . . . . . . . . 40

9.4 Large- $\boldsymbol{N}$ solution of nonminimal $\mathrm{CP}(\boldsymbol{N}-\mathbf{1}) \ldots \ldots \ldots$

9.4.1 Strong coupling phase with broken $Z_{N} \ldots \ldots \ldots$. . . 44

9.4.2 Coulomb/confining phase . . . . . . . . . . . 44

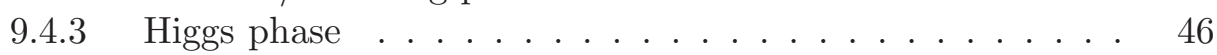

9.4 Goldstino . . . . . . . . . . . . . . . 46 
9.5 Large $N$ in nonminimal heterotic weighted

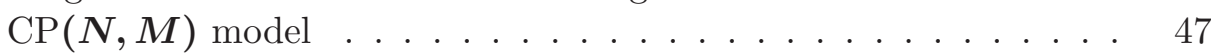

9.6 Large $\boldsymbol{N}$ in heterotic $\mathrm{O}(\boldsymbol{N})$ model . . . . . . . . . 48

10 In the uncharted waters 50

11 Conclusions

Acknowledgments

Appendix: Various representations of the nonminimal heterotic model

References 


\section{Part I \\ Introductory}

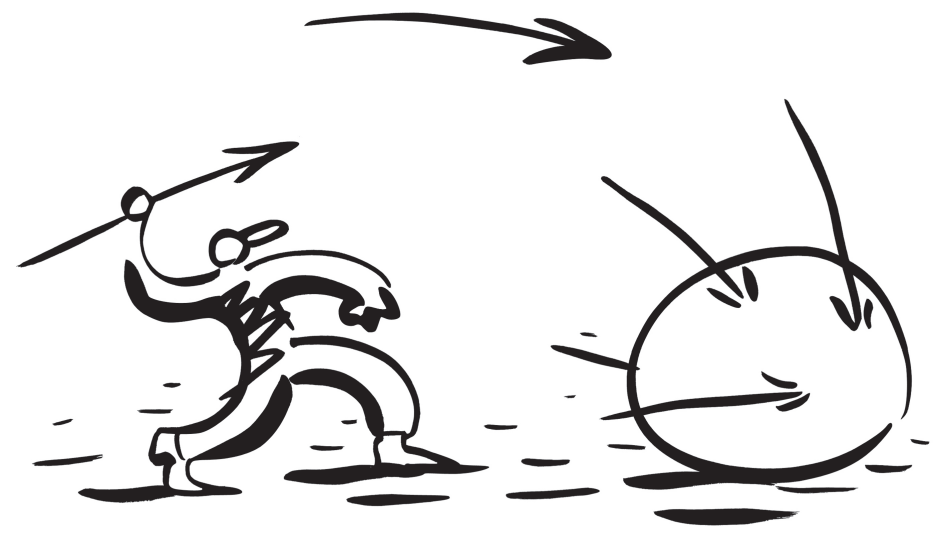




\section{Introduction}

Isaak Yakovlevich Pomeranchuk, the founder of the ITEP Theory Department, died in December of 1966, only six years before the advent of revolutionary changes in high-energy physics. His work with Landau [1] (see also [2]) on the so-called Moscow zero charge (currently known as infrared freedom in Abelian gauge theories), shaped the subsequent research on gauge theories which culminated in 1973, with the discovery of asymptotic freedom in non-Abelian gauge theories 3. Non-Abelian gauge theories proved to be the basis of the modern theory 2

Asymptotic freedom is just one aspect of these theories. Another aspect is a unique behavior in the infrared domain, at strong coupling, known as confinement, or, sometimes, color confinement. Despite four decades of vigorous efforts analytic understanding of the phenomenon of color confinement in quantum chromodynamics is still incomplete. At the same time significant advances occurred in 1994 when Seiberg and Witten solved $\mathcal{N}=2$ super-Yang-Mills theory [5].

In the mid-1970s Nambu, 't Hooft, and Mandelstam (independently) put forward an idea [6] of a "dual Meissner effect" as the underlying mechanism for color confinement. Within their conjecture, in appropriate YangMills theories chromomagnetic "monopoles" condense leading to formation of "chromoelectric flux tubes" between the probe quarks. At that time the Nambu-'t Hooft-Mandelstam paradigm was not even a physical scenario, rather a dream, since people had no clue as to the main building blocks such as non-Abelian flux tubes.

The Seiberg-Witten solution [5] triumphantly demonstrated the emergence of the confining strings as a result of a small $\mathcal{N}=1$ deformation $\mathcal{N}=2$ super-Yang-Mills theory.

However, although these strings appear in the non-Abelian theory they turned out to be Abelian in their structure [7], in essence identical to the Abrikosov-Nielsen-Olesen (ANO) strings [8].

Just like the fundamental string in string theory, the ANO string (at low excitation energies) is fully characterized by the position of its center in the perpendicular plane the so-called translational moduli. The orientation of the magnetic flux in the string core is rigidly fixed in the SW solution.

\footnotetext{
${ }^{2}$ Pomeranchuk witnessed the discovery of a two-dimensional asymptotically free field theory as early as in 1958 4, but at that time due attention was not paid to this work.
} 
Say, for the $\mathrm{SU}(2)$ gauge group it can be aligned along the third axis in the color space. Shortly after the SW discovery it was realized that for QCDlike theories, in which there are no preferred directions in the color space, it would be more appropriate to have the flux in the string core fluctuating freely "inside" the non-Abelian group. In other words for QCD strings it is desirable to have additional orientational moduli on the string world sheet. Such strings became known as non-Abelian.

The search for genuinely non-Abelian strings started in the end of 1990s and culminated in their discovery [9] in 2003. Dynamics of the extra orientational - moduli on the string world sheet was demonstrated to be described by $\mathrm{CP}(N-1)$ model, where $N$ is the number of colors in the bulk theory. Since then a large variety of non-Abelian strings became known; some of them support two-dimensional theories that had been known for decades, others exhibit nontrivial and largely unexplored sigma models on the string world sheet. This review is devoted to two-dimensional sigma models which came into the limelight in connection with the non-Abelian strings. The review is by necessity brief and represents, in a sense, a travel guide in this subject.

Historically nontrivial sigma models on the string world sheet first emerged in the context of supersymmetric bulk theories. Now it is clear that supersymmetry is not necessary, nonsupersymmetric bulk theories can support them too [10, 11]. Due to the fact that we will mainly focus on least explored world-sheet theories - heterotic two-dimensional sigma models - our discussion will be tied up with supersymmetry. A significant part of this review is devoted to results which we obtained after 2008. For a review before 2009 see [12].

\section{How world-sheet models appear: the simplest example}

The simplest and historically the first model supporting non-Abelian strings is [9] $\mathcal{N}=2$ super-Yang-Mills theory with the number of colors equal to the number of flavors (i.e. if the gauge group is $\mathrm{SU}(2)$, to which we will limit ourselves in this section, we introduce two (s)quark flavors). Moreover, we add a $\mathrm{U}(1)$ factor to the gauge group, so that, in fact, the gauge group is $\mathrm{U}(2)$. We endow this $\mathrm{U}(1)$ factor with the Fayet-Iliopoulos term $\xi$ [13]. The 
latter is needed to make non-Abelian strings BPS-saturated. BPS saturation is not a necessary condition. However, it simplifies calculations.

The bosonic part of the basic U(2) theory with two flavors has the form [9] (in the Euclidean space)

$$
\begin{aligned}
\mathcal{L} & =\frac{1}{4 g_{2}^{2}}\left(F_{\mu \nu}^{a}\right)^{2}+\frac{1}{4 g_{1}^{2}}\left(F_{\mu \nu}\right)^{2}+\frac{1}{g_{2}^{2}}\left|D_{\mu} a^{a}\right|^{2}+\frac{1}{g_{1}^{2}}\left|\partial_{\mu} a\right|^{2} \\
& +\left|\nabla_{\mu} q^{A}\right|^{2}+\left|\nabla_{\mu} \overline{\tilde{q}}^{A}\right|^{2}+V\left(q^{A}, \tilde{q}_{A}, a^{a}, a\right) .
\end{aligned}
$$

Here $D_{\mu}$ is the covariant derivative in the adjoint representation of $\mathrm{SU}(2)$, and

$$
\nabla_{\mu}=\partial_{\mu}-\frac{i}{2} A_{\mu}-i A_{\mu}^{a} T^{a}, \quad T^{a}=\frac{1}{2} \tau^{a},
$$

where $\tau^{a}$ are the Pauli matrices acting in the color $\mathrm{SU}(2)$ group. The coupling constants $g_{1}$ and $g_{2}$ correspond to the $\mathrm{U}(1)$ and $\mathrm{SU}(2)$ sectors, respectively. With our conventions, the $\mathrm{U}(1)$ charges of the fundamental matter fields are $\pm 1 / 2$. Two squark fields are denoted by $q^{A}$ and $\tilde{q}_{A}$, respectively (the flavor index $A=1,2$ ). The doubling of the (s)quark fields is required by $\mathcal{N}=2$ supersymmetry. In addition to the flavor index $A$ the the (s)quark fields carry $\mathrm{SU}(2)$ doublet index too; therefore, they can be viewed as a $2 \times 2$ matrix. Moreover, $a^{c}(c=1,2,3)$ is the complex scalar field in the adjoint representation of $\mathrm{SU}(2)$, the superpartner of the $\mathrm{SU}(2)$ gauge bosons, while $a$ without the superscript is the superpartner of the $\mathrm{U}(1)$ gauge boson. For brevity we will refer to these fields as to "adjoints."

The potential $V\left(q^{A}, \tilde{q}_{A}, a^{a}, a\right)$ in the Lagrangian (10) is a sum of $D$ and $F$ terms,

$$
\begin{aligned}
& V\left(q^{A}, \tilde{q}_{A}, a^{a}, a\right)=\frac{g_{2}^{2}}{2}\left(\frac{i}{g_{2}^{2}} \varepsilon^{a b c} \bar{a}^{b} a^{c}+\bar{q}_{A} T^{a} q^{A}-\tilde{q}_{A} T^{a} \overline{\tilde{q}}^{A}\right)^{2} \\
& +\frac{g_{1}^{2}}{8}\left(\bar{q}_{A} q^{A}-\tilde{q}_{A} \overline{\tilde{q}}^{A}\right)^{2}+2 g_{2}^{2}\left|\tilde{q}_{A} T^{a} q^{A}\right|^{2}+\frac{g_{1}^{2}}{2}\left|\tilde{q}_{A} q^{A}-\xi\right|^{2} \\
& +\frac{1}{2} \sum_{A=1}^{N}\left\{\left|\left(a+\sqrt{2} m_{A}+2 T^{a} a^{a}\right) q^{A}\right|^{2}+\left|\left(a+\sqrt{2} m_{A}+2 T^{a} a^{a}\right) \overline{\tilde{q}}^{A}\right|^{2}\right\} .
\end{aligned}
$$

Here $m_{A}$ are the (s)quark mass terms, and the sum over the repeated flavor indices $A=1,2$ is implied. 
Let us discuss the vacuum structure of this model. Nonvanishing of the Fayet-Iliopoulos term $\xi \neq 0$ implies an isolated vacuum with the maximal possible value of condensed (s)quarks - two. The vacua of the theory (1) are determined by the zeros of the potential (3). The adjoint fields develop the following vacuum expectation values (VEVs):

$$
\langle\Phi\rangle=-\frac{1}{\sqrt{2}}\left(\begin{array}{cc}
m_{1} & 0 \\
0 & m_{2}
\end{array}\right),
$$

where we defined the scalar adjoint matrix as

$$
\Phi \equiv \frac{1}{2} a+T^{a} a^{a} .
$$

If $m_{1}=m_{2}$ and $\xi=0$ the $\mathrm{SU}(2) \times \mathrm{U}(1)$ gauge group remains classically unbroken, since in this case $m$ can be absorbed in $a$. Alternatively, we can set $m=0$ from the beginning. However, if $m_{1} \neq m_{2} \mathrm{SU}(2)$ is broken down to $\mathrm{U}(1)$. Furthermore, if $\xi \neq 0$ we must take into account the squark VEVs which results in Higgsing of all gauge bosons..

In the vacuum the squark VEVs have a peculiar color-flavor locked form

$$
\left\langle q^{k A}\right\rangle=\left\langle\overline{\tilde{q}}^{k A}\right\rangle=\sqrt{\frac{\xi}{2}}\left(\begin{array}{ll}
1 & 0 \\
0 & 1
\end{array}\right), \quad k=1,2, \quad A=1,2 .
$$

The potential (3) vanishes if $\Phi$ and $q$ are chosen according to (44) and (6), respectively. ( $\xi$ is assumed to be large, $\xi \gg \Lambda^{2}$, to warrant quasi classical treatment.)

The vacuum field (6) results in the spontaneous breaking of both gauge and flavor SU(2)'s. A diagonal global SU(2) survives, however,

$$
\mathrm{U}(2)_{\text {gauge }} \times \mathrm{SU}(2)_{\text {flavor }} \rightarrow \mathrm{SU}(2)_{C+F} .
$$

Thus, a color-flavor locking takes place in the vacuum.

Why does the model described above support a novel type of strings, non-Abelian?

The conventional ANO string corresponds to a U(1) winding of the phase of all squark fields in the plane, perpendicular to the string axis,

$$
q^{k A} \longrightarrow \sqrt{\frac{\xi}{2}} e^{i \alpha}\left(\begin{array}{ll}
1 & 0 \\
0 & 1
\end{array}\right),
$$


where $\alpha$ is the polar angle in the perpendicular plane (see Fig. 11). Its topological stability is due to $\pi_{1}(\mathrm{U}(1))=Z$. Now we have more options, however, due to the fact that $\mathrm{SU}(2)_{C+F}$ has center. Usually people say that $\pi_{1}(\mathrm{SU}(2))$ is trivial and, therefore there are no other topologically stable strings.

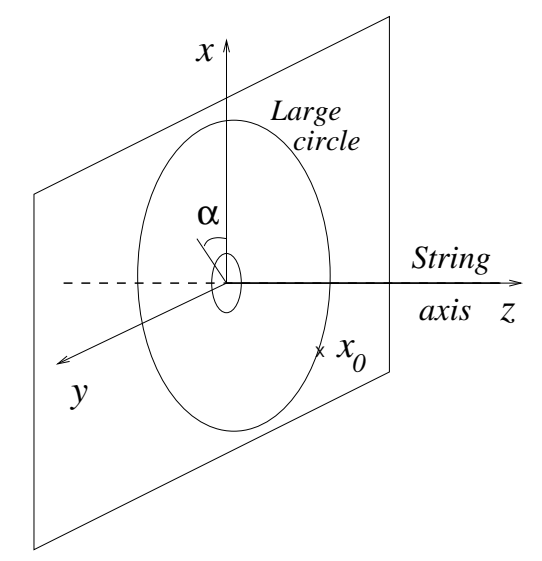

Figure 1: Geometry of the string.

This is not quite the case in the model at hand. Observe that the center of the $\mathrm{SU}(2)$ group, $Z_{2}$, belongs to the $\mathrm{U}(1)$ factor too. This means that we can split the $2 \pi$ windings in two halves: the first (from 1 to -1 ) is carried out in $\mathrm{U}(1)$, while the second, from -1 to 1 in $\mathrm{SU}(2)$ (e.g. around the third axis). This is clearly, a topologically stable configuration, albeit the stability is of the $Z_{2}$ type. Correspondingly, the winding ansatz takes the form

$$
q^{k A} \longrightarrow \sqrt{\frac{\xi}{2}}\left(\begin{array}{cc}
e^{i \alpha} & 0 \\
0 & 1
\end{array}\right) \quad \text { or } \quad q^{k A} \longrightarrow \sqrt{\frac{\xi}{2}}\left(\begin{array}{cc}
1 & 0 \\
0 & e^{i \alpha}
\end{array}\right)
$$

depending on whether we use the combination of generators $T_{\mathrm{U}(1)}+T_{\mathrm{SU}(2)}^{3}$ or $T_{\mathrm{U}(1)}-T_{\mathrm{SU}(2)}^{3}$.

It is clear, that the ansatz (9) breaks the color-flavor locked $\mathrm{SU}(2)$ down to $\mathrm{U}(1)$. The particular way of embedding is unimportant. Instead of $T^{3}$ we could have chosen any other generator of $\mathrm{SU}(2)$. In other words, the existence of the string (91) implies the existence of the whole family of strings parametrized by $\mathrm{SU}(2) / \mathrm{U}(1)$ moduli. The theory of the moduli fields on the string world sheet is the sigma model on the $\mathrm{SU}(2) / \mathrm{U}(1)$ coset space. This is the celebrated $\mathrm{CP}(1)$ model. It is asymptotically free in the UV and strongly 
coupled in the IR. Since the bulk theory has eight supercharges and the string is $1 / 2 \mathrm{BPS}$ saturated, the world-sheet model has four supercharges. In other words, its supersymmetry is $\mathcal{N}=(2,2)$. The existence of the orientational moduli means that the flux through the string does not have a preferred orientation inside $\mathrm{SU}(2)$. This is a genuinely non-Abelian string. Non-Abelian strings are formed if all non-Abelian bulk degrees of freedom participate are equally operative at the scale of string formation

Since the $\mathrm{CP}(1)$ model is equivalent to $\mathrm{O}(3)$ (see e.g. [14]), the orientational moduli can be represented as a unit vector (in the "isospace") attached to every point of the string and allowed to fluctuate freely see Fig. 2 .

If the tension of the ANO string is $4 \pi \xi$, the tension of the non-Abelian string is $2 \pi \xi$, in the $\mathrm{U}(2)$ bulk theory. Thus, the ANO string is, in a sense, composite.

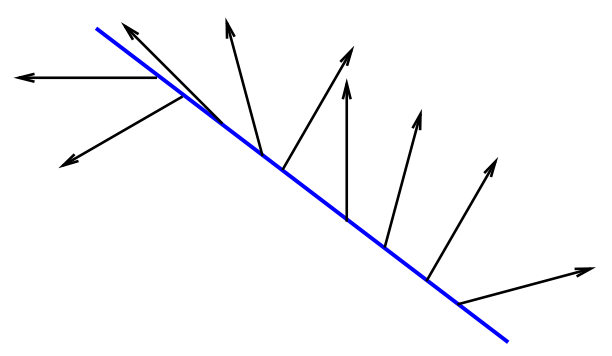

Figure 2: $\mathrm{O}(3)$ sigma model on the string world sheet.

For bulk theories with the $\mathrm{U}(N)$ gauge group the world sheet theory on the non-Abelian string is given by $\mathrm{CP}(N-1)$ models 9 .

\section{Supersymmetry in the bulk and BPS strings}

The degree of supersymmetry of BPS-saturated [15] non-Abelian strings is determined by supersymmetry in the bulk. Thus, $\mathcal{N}=2$ bulk theories support $\mathcal{N}=(2,2)$ models on their world sheet 9 , while $\mathcal{N}=0$ bulk theories give rise to non-supersymmetric non-Abelian strings (e.g. [10]). The most interesting type of strings - heterotic - appear in the $\mathcal{N}=1$ bulk theories [16, 17, 18]. In this case the world-sheet Lagrangian possesses $\mathcal{N}=(0,2)$ supersymmetry (it can be minimal or nonminimal), which is usually further spontaneously broken due to an appropriate Goldstino field on the world sheet. 
Nonsupersymmetric and $(2,2)$ supersymmetric two-dimensional sigma models are thoroughly studied, see e.g. the review [19]. As far as heterotic $(0,2)$ models are concerned, till recently only some general aspects have been discussed [20, 21, 22, 23, 24, 25]. However, emergence of these theories on the string world sheet gave a strong impetus for further studies, see e.g. [26, 27, 28, 29, 30, 31].

\section{Basic models}

In the vast majority of examples studied so far, the world-sheet theories on non-Abelian strings are various versions of $\mathrm{CP}(N-1)$ models: with or without twisted masses, with or without extra fields, with or without supersymmetry, and their extensions such as the so-called $z n$ and weighted $\operatorname{CP}(N, M)$ models. All these models have two (sometimes even three) distinct representations. In this section we will briefly discuss these representation using the simplest example: non-supersymmetric and (2.2) supersymmetric $\mathrm{CP}(N-1)$ without twisted mass.

\subsection{Geometric formulation}

A generic Lagrangian of any sigma-model with the Kählerian target space is

$$
\mathcal{L}_{\mathrm{CP}(N-1)}=G_{i \bar{j}} \partial^{\mu} \bar{\phi}^{\bar{j}} \partial_{\mu} \phi^{i}
$$

where $G_{i \bar{j}}$ is the Kähler metric,

$$
G_{i \bar{j}}=\frac{\partial^{2} K(\phi, \bar{\phi})}{\partial \phi^{i} \partial \bar{\phi}^{\bar{j}}}
$$

and $K(\phi, \bar{\phi})$ is the Kähler potential. For the $\mathrm{CP}(N-1)$ model one can choose the following Kähler potential:

$$
K=\frac{2}{g_{0}^{2}} \log \left(1+\sum_{i, \bar{j}=1}^{N-1} \bar{\phi}^{\bar{j}} \delta_{\bar{j} i} \phi^{i}\right),
$$

corresponding to the so-called round Fubini-Study metric. The bare coupling constant is denoted by $g_{0}^{2}$. 
It is not difficult to supersymmetrize the model (10) and (11). Its $\mathcal{N}=$ (2.2) generalization can be written as [32]

$$
\begin{aligned}
\mathcal{L}_{\mathcal{N}=(2,2)}=\int d^{4} \theta K(\Phi, \bar{\Phi}) & =G_{i \bar{j}}\left[\partial^{\mu} \bar{\phi}^{\bar{j}} \partial_{\mu} \phi^{i}+i \bar{\psi}^{\bar{j}} \gamma^{\mu} \mathcal{D}_{\mu} \psi^{i}\right] \\
& -\frac{1}{2} R_{i \bar{j} \bar{k}(\bar{\psi}}\left(\bar{j} \psi^{i}\right)\left(\bar{\psi}^{\bar{l}} \psi^{k}\right),
\end{aligned}
$$

where $\Phi^{i}$ and $\bar{\Phi}$ are the chiral and antichiral superfields

$$
\Phi^{i}\left(x^{\mu}+i \bar{\theta} \gamma^{\mu} \theta\right), \quad \bar{\Phi}^{\bar{j}}\left(x^{\mu}-i \bar{\theta} \gamma^{\mu} \theta\right)
$$

of which the lowest components are $\phi^{i}$ and $\bar{\phi}$ (see e.g. [14]), $R_{i \bar{j} k \bar{l}}$ is the Riemann tensor,

$$
\mathcal{D}_{\mu} \psi^{i}=\partial_{\mu} \psi^{i}+\Gamma_{k l}^{i} \partial_{\mu} \phi^{k} \psi^{l}
$$

is the covariant derivative, $\Gamma_{k l}^{i}$ are the Christoffel symbols, and we use the notation $\bar{\theta}=\theta^{\dagger} \gamma^{0}, \bar{\psi}=\psi^{\dagger} \gamma^{0}$ for the fermion objects. The $\gamma$ matrices are chosen as

$$
\gamma^{0}=\gamma^{t}=\sigma_{2}, \quad \gamma^{1}=\gamma^{z}=i \sigma_{1}, \quad \gamma_{5} \equiv \gamma^{0} \gamma^{1}=\sigma_{3} .
$$

For $\mathrm{CP}(N-1)$ target space, as for any symmetric manifold, the Ricci-tensor $R_{i \bar{j}}$ is proportional to the metric, see Eq. (18) below. Both versions of this model - supersymmetric and non-supersymmetric are asymptotically free [33]. In the former case the $\beta$ function is one-loop exact,

$$
\beta_{\mathcal{N}=(2,2)} \equiv \frac{\partial g_{0}^{2}}{\partial \log M_{\mathrm{uv}}}=-\frac{g^{4} N}{4 \pi} .
$$

Only bosons contribute at first loop. In non-supersymmetric $\mathrm{CP}(N-1)$ model [34, 14]

$$
\beta_{\mathrm{CP}(N-1)}=-\frac{g^{4} N}{4 \pi}\left(1+\frac{g^{2}}{2 \pi}+\ldots\right),
$$

where ellipses stand for the third and higher loops.

For completeness, concluding this section let us add a few extra useful expressions,

$$
G_{i \bar{j}}=\frac{2}{g^{2}}\left(\frac{\delta_{i \bar{j}}}{\chi}-\frac{\bar{\phi}^{i} \phi^{\bar{j}}}{\chi^{2}}\right), \quad G^{i \bar{j}}=\frac{g^{2}}{2} \chi\left(\delta^{i \bar{j}}+\phi^{i} \bar{\phi}^{\bar{j}}\right),
$$




$$
\begin{array}{ll}
\Gamma_{k l}^{i}=-\frac{\delta_{k}^{i} \bar{\phi}^{\bar{l}}+\delta_{l}^{i} \bar{\phi}^{\bar{k}}}{\chi}, & \Gamma_{\bar{k} \bar{l}}^{\bar{i}}=-\frac{\delta_{\bar{k}}^{\bar{i}} \phi^{l}+\delta_{\bar{l}}^{\bar{i}} \phi^{k}}{\chi}, \\
R_{i \bar{j} k \bar{l}}=-\frac{g^{2}}{2}\left(G_{i \bar{j}} G_{k \bar{l}}+G_{k \bar{j}} G_{i \bar{l}}\right), & R_{i \bar{j}}=-G^{k \bar{j}} R_{i \bar{j} k \bar{l}}=\frac{g^{2} N}{2} G_{i \bar{j}}, \\
\chi \equiv 1+\sum_{m}^{N-1} \bar{\phi}^{\bar{m}} \phi^{m} . &
\end{array}
$$

\subsection{Gauged formulation}

An alternative formulation - the so-called gauged formulation - was suggested by Witten [35, 36]. Being completely equivalent to the geometric formulation it is more convenient for the large- $N$ solution of the model.

The $\mathrm{CP}(N-1)$ target space is the coset $\mathrm{SU}(N) /(\mathrm{SU}(N-1) \times \mathrm{U}(1)$. In the gauged formulation we build the Lagrangian $\mathcal{L}_{\mathrm{CP}(N-1)}$ starting from an $N$-plet of complex bosonic fields $n^{i}$ where $i=1,2, \ldots, N$. The fields $n^{i}$ are scalar (i.e. spin-0), and are subject to the constraint

$$
\bar{n}_{i} n^{i}=1
$$

The Lagrangian takes the form

$$
\mathcal{L}_{\mathrm{CP}(N-1)}=\frac{2}{g^{2}}\left|\mathcal{D}_{\mu} n^{i}\right|^{2}-D\left(n_{i}^{\dagger} n^{i}-1\right)
$$

where the covariant derivative $\mathcal{D}_{\mu}$ is defined as

$$
\mathcal{D}_{\mu} n^{i} \equiv\left(\partial_{\mu}-i A_{\mu}\right) n^{i}
$$

Here $A_{\mu}$ is an auxiliary vector field implementing $\mathrm{U}(1)$ gauge invariance, while $D$ is an auxiliary real scalar field implementing the constraint (19). Neither $A_{\mu}$ nor $D$ have kinetic terms in the Lagrangian (20).

Sometimes it is convenient to rescale the $n$ and $D$ fields as follows:

$$
\begin{aligned}
\mathcal{L}_{\mathrm{CP}(N-1)} & =\left|\mathcal{D}_{\mu} n^{i}\right|^{2}-D\left(\bar{n}_{i} n^{i}-2 \beta\right), \\
\beta & \equiv \frac{1}{g^{2}},
\end{aligned}
$$

making the kinetic term canonic. The vacuum expectation value of $D$ will then play the role of the $n$-field mass squared. 
From Sec. 4.1 we see that the $\operatorname{CP}(N-1)$ target space is parametrized by $2 N-2$ real degrees of freedom. There are $2 N$ real degrees of freedom in the $n^{i}$ fields. The constraint (19) reduces this number to $2 N-1$, while the $\mathrm{U}(1)$ gauge invariance further reduces it to $2 N-2$.

The fields $\phi^{i}$ of the geometric formulation can be related to $n^{i}$ (on a particular patch) by singling out one of the components of $n^{i}$, say, $n^{N}$, and defining

$$
\phi^{i}=\frac{n^{i}}{n^{N}}, \quad i=1,2, \ldots, N-1
$$

The easiest way to extend the above formalism to $\mathcal{N}=(2,2)$ supersymmetry is to start from $\mathcal{N}=1$ SQED in four dimensions with $N$ flavors of chiral matter superfields (with one and the same charge), plus the FayetIliopoulos term $\tilde{\xi}$,

$$
\mathcal{L}=\left\{\frac{1}{4 e^{2}} \int d^{2} \theta W^{2}+\text { H.c. }\right\}+\int d^{4} \theta \sum_{i=1}^{N}\left(\bar{Q}_{i} e^{V} Q^{i}\right)-\tilde{\xi} \int d^{4} \theta V,
$$

where

$$
Q^{i}=n^{i}+\sqrt{2} \theta \xi^{i}+\theta^{2} F^{i}
$$

This theory does not exist in four dimensions due to the chiral anomaly. However, we will use it only as a starting point, with the intention of reducing it to two dimensions. In two dimensions it becomes well-defined. The $\mathcal{N}=$ $(2,2) \mathrm{CP}(N-1)$ model is obtained in the limit $e^{2} \rightarrow \infty$. In this limit both the photon and photino kinetic terms can be dropped, and we obtain (in components)

$$
\begin{aligned}
\mathcal{L}_{\mathcal{N}=(2,2)} & =\left|\mathcal{D}_{\mu} n^{i}\right|^{2}-2|\sigma|^{2}\left|n^{i}\right|^{2}-D\left(\left|n^{i}\right|^{2}-2 \beta\right) \\
& +\bar{\xi}_{j R} i \mathcal{D}_{L} \xi_{R}^{j}+\bar{\xi}_{j L} i \mathcal{D}_{R} \xi_{L}^{j} \\
& +\left[\sqrt{2} \sigma \bar{\xi}_{j R} \xi_{L}^{j}+\sqrt{2} \bar{n}_{j}\left(\lambda_{R} \xi_{L}^{j}+\lambda_{L} \xi_{R}^{j}\right)+\text { H.c. }\right] .
\end{aligned}
$$

Here $\sigma=\left(A_{x}+i A_{y}\right) / \sqrt{2}$ is a part of the superfield $V$ (in the Wess-Zumino gauge), along with $A_{t, z}, D$, and $\lambda_{R, L}$ (for geometrical conventions see Fig. 1). All these fields enter in the Lagrangian (25) without kinetic terms. The latter will be generated, however, at one-loop level, dynamically. The spinor fields $\lambda_{R, L}$ implement the constraint $\bar{n}_{j} \xi^{j}=0$. The constraint $\left|n^{i}\right|^{2}=2 \beta$ is implemented by the auxiliary $D$ field. The covariant derivative is defined in 
(21). Finally, the Fayet-Iliopoulos term in (24) is related to $2 \beta$, namely, $\tilde{\xi} \rightarrow$ $2 \beta$ (note that in two dimensions the Fayet-Iliopoulos term is dimensionless).

\subsection{CP(1): a special case}

The case $N=2$, when we deal with the $\mathrm{CP}(1)$ model, is special. Indeed, the $\mathrm{CP}(1)$ target space is isomorphic to $\mathrm{O}(3)$, implying that the $\mathrm{CP}(1)$ model can be formulated in terms of a triplet of real fields $S^{a}$. The $\mathrm{O}(3)$ model, in turn, opens the series of the $\mathrm{O}(N)$ models. For $N>3$ the $\mathrm{O}(N)$ target space is not Kählerian. Thus, supersymmetrization of the $\mathrm{O}(N)$ models with $N>3$ results in $\mathcal{N}=(1,1)$ supersymmetry.

To explicitly pass from $\mathrm{CP}(1)$ to $\mathrm{O}(3)$ one needs expressions relating the $\vec{S}$ fields to the $n^{i}$ fields. Given the fact that in this case $n^{i}$ s are spinors of $\mathrm{SU}(2)$ while $\vec{S}$ is the $\mathrm{O}(3)$ vector one can write

$$
S^{a}=\bar{n} \tau^{a} n, \quad a=1,2,3,
$$

where $\tau^{a}$ are the Pauli matrices which satisfy the Fierz transformation formula

$$
\vec{\tau}_{\alpha \beta} \vec{\tau}_{\delta \gamma}=2 \delta_{\alpha \gamma} \delta_{\delta \beta}-\delta_{\alpha \beta} \delta_{\delta \gamma}
$$

Making use of (27) one concludes that

$$
\vec{S}^{2}=(\bar{n} n)^{2}=1 \text {. }
$$

Thus,

$$
\mathcal{L}_{\mathrm{O}(3)}=\frac{1}{2 g^{2}} \partial_{\mu} S^{a} \partial^{\mu} S^{a}, \quad S^{a} S^{a}=1, \quad a=1,2,3 .
$$

Supergeneralization of (29) is straightforward [37]. One introduces a triplet of real superfields $\sigma^{a}(x, \theta)$,

$$
N^{a}(x, \theta)=S^{a}+\bar{\theta} \chi^{a}+\frac{1}{2} \bar{\theta} \theta F^{a}
$$

where $\vec{S}$ and $\vec{F}$ are bosonic fields while $\vec{\chi}$ denotes two-component Majorana fields (the $\theta$ coordinate is also Majorana).

Then the supersymmetric Lagrangian takes the form

$$
\begin{aligned}
\mathcal{L}_{\mathrm{O}(3)} & =\frac{1}{g^{2}} \int d^{2} \theta\left(\frac{1}{2} \bar{D}_{\alpha} N^{a} D_{\alpha} N^{a}\right) \\
& =\frac{1}{2 g^{2}}\left\{\partial^{\mu} S^{a} \partial_{\mu} S^{a}+\frac{i}{2} \bar{\chi}^{a} \gamma^{\mu} \stackrel{\leftrightarrow}{\partial}_{\mu} \chi^{a}+\vec{F}^{2}\right\} .
\end{aligned}
$$


with the constraint

$$
N^{a}(x, \theta) N^{a}(x, \theta)=1,
$$

which replaces the nonsupersymmetric version of this constraint $\vec{S}^{2}=1$.

Decomposing (32) in components we get

$$
\vec{S}^{2}=1, \quad \vec{S} \vec{\chi}=0, \quad \vec{F} \vec{S}=\frac{1}{2}\left(\bar{\chi}^{a} \chi^{a}\right) .
$$

As usual, the $F$ term enters with no derivatives. Eliminating $F$ by virtue of the equations of motion one obtains [37, 38]

$$
\mathcal{L}=\frac{1}{2 g^{2}}\left\{\partial^{\mu} S^{a} \partial_{\mu} S^{a}+\frac{i}{2} \bar{\chi}^{a} \gamma^{\mu} \stackrel{\leftrightarrow}{\partial}_{\mu} \chi^{a}+\frac{1}{4}\left(\bar{\chi}^{a} \chi^{a}\right)^{2}\right\},
$$

plus the first two constraints in Eq. (33).

The global $\mathrm{O}(3)$ symmetry is explicit in this Lagrangian. Moreover, $(1,1)$ supersymmetry is built in. In fact, supersymmetry of this model is $\mathcal{N}=(2,2)$ due to the Kählerian nature of the target space 2-sphere [39].

A special nature of the $\mathrm{CP}(1)$ target space manifests itself in the fact that for the $\mathrm{CP}(N-1)$ with $N>2$ minimal heterotic $(0,2)$ models do not exist [40], while it does exists for $\mathrm{CP}(1)$, see Sec. 9.2.1.

\section{$5 \quad$ Witten's large- $N$ solution}

In this section we will briefly discuss large- $N$ solutions of the simplest twodimensional models emerging on the world sheet of non-Abelian strings. Massless non-supersymmetric and $\mathcal{N}=(2,2)$ supersymmetric $\mathrm{CP}(N-1)$ models were solved at large $N$ by Witten [35].

\subsection{Non-supersymmetric $\operatorname{CP}(N-1)$}

Let us turn to (22) rescaling the coupling constant à la 't Hooft to make explicit the $N$ dependence,

$$
\mathcal{L}_{\mathrm{CP}(N-1)}=\left(\partial_{\alpha}+i A_{\alpha}\right) \bar{n}_{i}\left(\partial^{\alpha}-i A^{\alpha}\right) n^{i}-D\left(\bar{n}_{i} n^{i}-\frac{N}{\lambda}\right)^{2},
$$

where

$$
\lambda \equiv \frac{g^{2} N}{2} .
$$


First, we study the vacuum structure of this model. Note that the Lagrangian (35) is quadratic in the $n^{i}$ fields; therefore these fields can be integrated out,

$$
Z=\int \mathcal{D} A_{\alpha} \mathcal{D} D \exp \left\{-N \operatorname{Tr} \ln \left[-\left(\partial_{\alpha}-i A_{\alpha}\right)^{2}-D\right]+i \frac{N}{\lambda} \int d^{2} x D\right\} .
$$

The Lorentz invariance of the theory tells us that if the saddle point exists it must be achieved at an $x$ independent value of $D$. Hence we can treat $D$ as a constant, vary with respect to $D$, and require the result to vanish. The same Lorentz invariance tells us that at the saddle point $A_{\alpha}=0$. In this way we arrive at the following equation:

$$
\frac{i}{\lambda}+\int \frac{d^{2} k}{4 \pi^{2}} \frac{1}{k^{2}-D}=0, \quad \frac{1}{\lambda}-\frac{1}{4 \pi} \log \frac{M_{\mathrm{uv}}^{2}}{D} .
$$

The integral in (38) is logarithmic and diverges in the ultraviolet, therefore we cut it off at $M_{\mathrm{uv}}^{2}$. In this way, starting from (38), we arrive at the equation

$$
D_{\mathrm{vac}} \equiv m^{2}=M_{\mathrm{uv}}^{2} e^{-4 \pi / \lambda}=M_{\mathrm{uv}}^{2} e^{-8 \pi / N g^{2}} \equiv \Lambda^{2} \text {. }
$$

The assumption of existence of the saddle point is confirmed a posteriori. The $n$-quanta mass $m$ is a physical parameter. Therefore, the right-hand side of (39) is renormalization-group invariant, $\Lambda^{2}$, the dynamical scale parameter of the $\mathrm{CP}(N-1)$ model. This is in full agreement with the first coefficient of the $\beta$ function in (17). The second coefficient is invisible to the leading order in $1 / N$.

Integrating the second equation in (38) over $D$ one readily reconstructs the effective potential as a function of $D$,

$$
V_{\text {eff }}=\frac{N}{4 \pi} D \log \frac{D}{e m^{2}}, \quad e=2.718 \ldots
$$

From the large- $N$ solution one can see that the constraint $\bar{n}_{i} n^{i}=1$ is lifted and the massive $n$ particles form a full $N$-plet. The $n$-mass is given in (39). Moreover, it is not difficult to see that the field $A_{\mu}$ acquires kinetic terms and become dynamical. Expanding the effective action (37) around the saddle point, one can easily check that cubic and higher orders in $D$ and $A$ are suppressed by powers of $1 / \sqrt{N}$. The linear term of expansion vanish. This is the essence of Eq. (38). We will focus on the quadratic terms of expansion. 


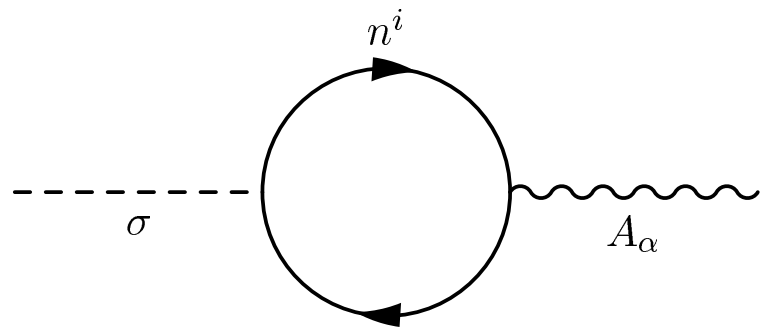

Figure 3: The vanishing of the $D A_{\alpha}$ mixing term in the effective Lagrangian.

It is not difficult to check (see e.g. [14]) that the cross term of the $D A$ type also vanish (see Fig. 3). Therefore, we need only consider the terms quadratic in $A$, see Fig. 4. A straightforward computation yields for the $A^{2}$ term 3

$$
\frac{N}{12 \pi m^{2}}\left(-g_{\mu \nu} k^{2}+k_{\mu} k_{\nu}\right)\left(1+O\left(k^{2} / m_{n}^{2}\right)\right) .
$$

This expression is automatically transversal, as expected given the $\mathrm{U}(1)$ gauge invariance of (35). The $O\left(k^{2}\right)$ term in (41) represents the standard kinetic term $F_{\mu \nu}^{2}$ of the photon field, more exactly,

$$
-\frac{N}{48 \pi m^{2}} F_{\mu \nu} F^{\mu \nu}
$$

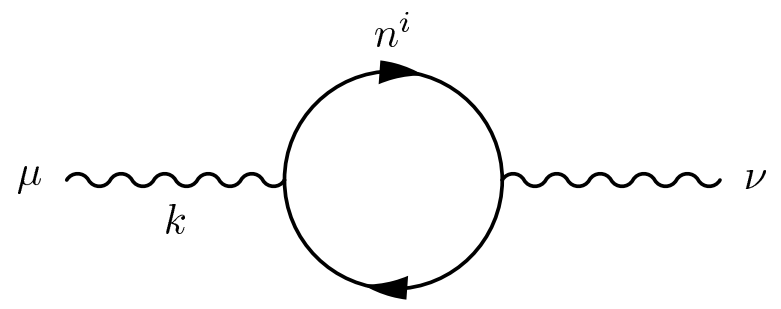

Figure 4: $O\left(A^{2}\right)$ terms in the effective Lagrangian.

It is convenient to rescale the $A$ field to make its kinetic term (42) canonically normalized. Upon this rescaling the effective Lagrangian takes the form

$$
\mathcal{L}_{\mathrm{eff}}=-\frac{1}{4} F_{\mu \nu}^{2}+\left(\partial_{\alpha}+i e_{n} A_{\alpha}\right) \bar{n}_{i}\left(\partial^{\alpha}-i e_{n} A^{\alpha}\right) n^{i}-m^{2} \bar{n}_{i} n^{i}
$$

\footnotetext{
${ }^{3}$ The $O\left(k^{4}\right), O\left(k^{4}\right)$, and so terms can be ignored since they have no impact on the position of the pole at $k^{2}=0$ of the photon Green's function.
} 
where the electric charge of the $n$ quanta $e_{n}$ is

$$
e_{n} \equiv m \sqrt{\frac{12 \pi}{N}}
$$

It has dimension of mass, which is the correct dimension of the electric charge in two-dimensional theories. Moreover, one should stress that at large $N$ the electric charge becomes small, $e_{n} / m \ll 1$, which implies, in turn, weak coupling.

Emergence of the massless gauge U(1) field ensures the presence of the Coulomb potential between charges states. In two dimensions static Coulomb potential is a linear rising potential. It leads to the confinement of kinks which carry electric charge [35]. Therefore this phase of the theory is called Coulomb/confining phase.

\subsection{Supersymmetric $\mathrm{CP}(N-1)$}

It is easy to generalize the large- $N$ solution of Sec. 5.1 to $\mathcal{N}=(2,2)$ model [35, 36]. The Lagrangian (25) is quadratic in both, the $n$ fields and their fermion superpartners $\xi$. Therefore, they can be integrated out exactly. Note that the auxiliary fields $A, \sigma$ and $\lambda_{L, R}$ form a supermultiplet. Hence, it is sufficient to find the kinetic term and mass for one of them in order to determine them all, provided that supersymmetry is unbroken. As we will see momentarily, it is indeed unbroken.

As in (38) we set $A_{\mu}=0$, and then integrate out $n^{i}$ and $\xi^{i}$. This yields

$$
\frac{\operatorname{Det}\left(-\partial_{\alpha}^{2}-2|\sigma|^{2}\right)^{N}}{\operatorname{Det}\left(-\partial_{\alpha}^{2}-D-2|\sigma|^{2}\right)^{N}}
$$

The denominator comes from the boson loop while the numerator from the fermion loop. It is obvious that supersymmetric vacuum (with $E_{\mathrm{vac}}=0$ ) is attained at $D=0$, when the ratio of the determinants in (45) reduces to unity.

The above conclusion is confirmed by an explicit calculation of the effective potential, the analog of (40),

$$
V_{\mathrm{eff}}=\frac{N}{4 \pi}\left[\left(D+2|\sigma|^{2}\right) \log \frac{D+2|\sigma|^{2}}{m^{2}}-D-2|\sigma|^{2} \log \frac{2|\sigma|^{2}}{m^{2}}\right],
$$


where we carried out renormalization using the analog of (39),

$$
2\left|\sigma_{\mathrm{vac}}\right|^{2} \equiv m^{2}=M_{\mathrm{uv}}^{2} e^{-8 \pi / N g^{2}} .
$$

The vacuum values of $D$ and $|\sigma|$ are obtained through minimization, i.e. by differentiating $V_{\text {eff }}$ in (46) over $D$ and $2|\sigma|^{2}$,

$$
\begin{aligned}
& \log \frac{D+2|\sigma|^{2}}{m^{2}}=0, \\
& \log \frac{D+2|\sigma|^{2}}{m^{2}}-\log \frac{2|\sigma|^{2}}{m^{2}}=0 .
\end{aligned}
$$

As was mentioned, the mass of the $\xi$ field is the same as as that of $n$, due to supersymmetry.

The kinetic term of the $A_{\mu}$ field and its superpartners is dynamically generated in much the same way as in Sec. 5.1. A crucial difference is that now the photon field $A_{\mu}$ acquires a nonvanishing (albeit small) mass through the Schwinger mechanism: the massless fermion loop shifts the pole in the photon propagator away from zero. This was noted already in 1979 [35. Needless to say, all superpartners of the photon field receive the same mass.

Consequences of massless vs. massive photon in two dimensions are radically different. Massless photons in two-dimensions (non-supersymmetric $\mathrm{CP}(N-1))$ lead to confinement of charged particles, while massive photons (supersymmetric $\mathrm{CP}(N-1))$ do not confine. In one-to-one correspondence with this is the existence of $N$ degenerate vacua in the non-confining case. In the confining case (i.e. massless photon) one of these vacua remains genuine while the remaining $N-1$ are uplifted and become quasistable states.

\section{Twisted masses}

The so-called twisted masses is the only mass deformation of the $\mathcal{N}=(2,2)$ model which preserves supersymmetry. The essence of this deformation is as follows 41. One starts from four-dimensional $\mathrm{CP}(N-1)$ model and couples $N-1$ conserved $\mathrm{U}(1)$ currents of this model to background gauge four-potential $A_{\mu}$. Then one reduces the model to two dimensions $t$ and $z$ simultaneously declaring the background fields $A_{x}$ and $A_{y}$ (Fig. 1) to be nonvanishing constants. The twisted masses $\mu$ and $\bar{\mu}$ are proportional to $A_{x} \pm i A_{y}$. 
In the geometric formulation of Sec. 4.1 the formal procedure can be described as follows. The theory (10) can be interpreted as an $\mathcal{N}=1$ theory of $N-1$ chiral superfields in four dimensions. The theory possesses $N-1$ $\mathrm{U}(1)$ isometries parametrized by $t^{a}, a=1, \ldots, N-1$. The Killing vectors of the isometries can be expressed via derivatives of the Killing potentials $D^{a}\left(\phi, \phi^{\dagger}\right)$,

$$
\frac{d \phi^{i}}{d t_{a}}=-i G^{i \bar{j}} \frac{\partial D^{a}}{\partial \bar{\phi}^{\bar{j}}}, \quad \frac{d \bar{\phi}^{\bar{j}}}{d t_{a}}=i G^{i \bar{j}} \frac{\partial D^{a}}{\partial \phi^{i}} .
$$

This defines the $\mathrm{U}(1)$ Killing potentials up to additive constants.

The isometries are evident from the expression (11) for the Kähler potential,

$$
\delta \phi^{i}=-i \delta t_{a}\left(T^{a}\right)_{k}^{i}(\phi)^{k}, \quad \delta \bar{\phi}^{\bar{j}}=i \delta t_{a}\left(T^{a}\right)_{\bar{l}}^{\bar{j}} \bar{\phi}^{\bar{l}}, \quad a=1, \ldots, N-1,
$$

(together with the similar variation of fermionic fields), where the generators $T^{a}$ have a simple diagonal form,

$$
\left(T^{a}\right)_{k}^{i}=\delta_{a}^{i} \delta_{k}^{a}, \quad a=1, \ldots, N-1 .
$$

The explicit form of the Killing potentials $D^{a}$ in $\mathrm{CP}(N-1)$ with the FubiniStudy metric is

$$
D^{a}=\frac{2}{g_{0}^{2}} \frac{\bar{\phi} T^{a} \phi}{1+\bar{\phi} \phi}, \quad a=1, \ldots, N-1 .
$$

Here we use the matrix notation implying that $\phi$ is a column $\phi^{i}$ and $\bar{\phi}$ is a row $\bar{\phi}^{\bar{j}}$.

The isometries allow us to introduce an interaction with $N-1$ distinct background U(1) gauge superfields $V_{a}$ by modifying the Kähler potential (11) in a gauge invariant way,

$$
K(\Phi, \bar{\Phi}) \rightarrow \tilde{K}(\Phi, \bar{\Phi}, V)=\frac{2}{g_{0}^{2}} \log \left(1+\bar{\Phi} \mathrm{e}^{V_{a} T^{a}} \Phi\right)
$$

where

$$
V_{a}=-\mu_{a} \bar{\theta}\left(1+\gamma_{5}\right) \theta-\bar{\mu}_{a} \bar{\theta}\left(1-\gamma_{5}\right) \theta .
$$

Thus, in our notation the complex masses $m_{a}$ are linear combinations of the constant $\mathrm{U}(1)$ gauge potentials,

$$
m_{a}=A_{y}^{a}+i A_{x}^{a}, \quad \bar{m}_{a}=m_{a}^{*}=A_{y}^{a}-i A_{x}^{a} .
$$


Passing to two dimensions we assume, of course, that there is no dependence on $x$ and $y$ in the chiral fields. It gives us the Lagrangian with the twisted masses included [41],

$$
\begin{aligned}
\mathcal{L}_{m}=\int d^{4} \theta K_{m}\left(\Phi, \Phi^{\dagger}, V\right) & =G_{i \bar{j}} g_{M N}\left[\mathcal{D}^{M} \phi^{\dagger \bar{j}} \mathcal{D}^{N} \phi^{i}+i \bar{\psi}^{\bar{j}} \gamma^{M} D^{N} \psi^{i}\right] \\
& -\frac{1}{2} R_{i \bar{j} k \bar{l}}\left(\bar{\psi}^{\bar{j}} \psi^{i}\right)\left(\bar{\psi}^{\bar{l}} \psi^{k}\right)
\end{aligned}
$$

where summation over $M$ includes, besides $M=\alpha=0,1$, also $M=+,-$. The metric $g_{M N}$ and extra $\gamma$ matrices are

$$
g_{M N}=\left(\begin{array}{rrrr}
1 & 0 & 0 & 0 \\
0 & -1 & 0 & 0 \\
0 & 0 & 0 & -\frac{1}{2} \\
0 & 0 & -\frac{1}{2} & 0
\end{array}\right), \quad \gamma^{+}=-i\left(1+\gamma_{5}\right), \quad \gamma^{-}=i\left(1-\gamma_{5}\right) .
$$

The gamma-matrices satisfy the following algebra:

$$
\bar{\Gamma}^{M} \Gamma^{N}+\bar{\Gamma}^{N} \Gamma^{M}=2 g^{M N},
$$

where the set $\bar{\Gamma}^{M}$ differs from $\Gamma^{M}$ by interchanging of the,+- components, $\bar{\Gamma}^{ \pm}=\Gamma^{\mp}$. The gauge covariant derivatives $\mathcal{D}^{M}$ are defined as

$$
\begin{aligned}
& \mathcal{D}^{\alpha} \phi=\partial^{\alpha} \phi, \quad \mathcal{D}^{\alpha} \bar{\phi}=\partial^{\alpha} \bar{\phi}, \\
& \mathcal{D}^{+} \phi=-\bar{\mu}_{a} T^{a} \phi, \quad \mathcal{D}^{-} \phi=\mu_{a} T^{a} \phi, \\
& \mathcal{D}^{+} \bar{\phi}=\bar{\phi} T^{a} \bar{\mu}_{a}, \quad \mathcal{D}^{-} \bar{\phi}=-\bar{\phi} T^{a} \mu_{a},
\end{aligned}
$$

and similarly for $\mathcal{D}^{M} \psi$, while the general covariant derivatives $D^{M} \psi$ 's are

$$
D^{M} \psi^{i}=\mathcal{D}^{M} \psi^{i}+\Gamma_{k l}^{i} \mathcal{D}^{M} \phi^{k} \psi^{l} .
$$

In the geometrical formulation we have $N-1$ complex twisted mass parameters. Introduction of the twisted masses in the gauged formulation will be discussed in Part II, Secs. 7.1 and 7.2. In the gauged formulation there are $N$ complex twisted mass parameters $m_{i}$ related to $\mu^{a}$,

$$
\mu^{i}=m_{i}-m_{N}, \quad i=1,2, \ldots, N-1 .
$$


(see (23) ) and subject to the constraint

$$
\sum_{i=1}^{N} m_{i}=0 .
$$

One of our tasks in what follows is the study of the phase diagram of the twodimensional model on the string world sheet. To this end it is convenient to have a discrete symmetry. A $Z_{N}$ symmetry is guaranteed if the mass parameters are adjusted as

$$
m_{j}=m_{0} \exp \left(\frac{2 \pi i j}{N}\right), \quad j=1,2, \ldots, N .
$$

Such a choice is referred to as $Z_{N}$ symmetric. It is always assumed in what follows if not stated to the contrary.

Note that $m_{0}$ can be chosen to be real and positive. Then $m_{N}$ is real and positive too. Alternatively, if $m_{0}=\left|m_{0}\right| \exp (-2 \pi i / N)$, then $m_{1}$ is real and positive. 


\section{Part II \\ Travel Guide}

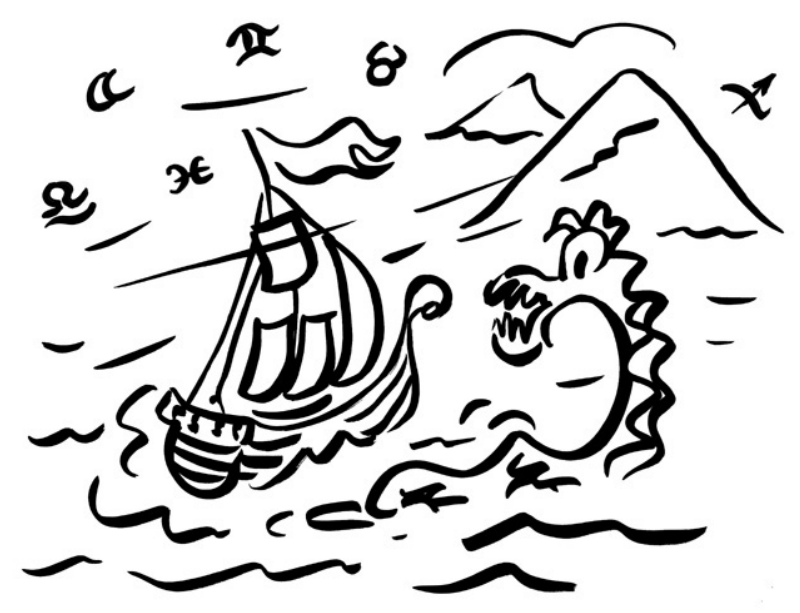

In Part II we will use Euclidean conventions. The Euclidean action reduces to the energy functional for static fields. 


\section{$7 \quad$ Large- $N$ solutions with twisted masses}

In this section we will briefly review those two-dimensional sigma models that are in the limelight ever since the discovery of the non-Abelian strings.

\section{1 $\mathrm{CP}(N-1)$ with $Z_{N}$ symmetric masses}

As a world-sheet model in the non-supersymmetric context, $\operatorname{CP}(N-1)$ with twisted masses was discussed in [10], and its large- $N$ solution in the $Z_{N}$ symmetric case was found in 42].

In the gauged formulation the Lagrangian has the form

$$
\mathcal{L}=\left|\mathcal{D}_{\alpha} n^{i}\right|^{2}+D\left(\left|n^{i}\right|^{2}-2 \beta\right)+\sum_{i=1}^{N}\left|\left(\sigma-m_{i}\right) n^{i}\right|^{2}
$$

were $\mathcal{D}_{\alpha}=\partial_{\alpha}-i A_{\alpha}$, the mass parameters $m_{i}$ are defined in Eq. (63), and $2 \beta$ is the bare coupling constant, see Eq. (22).

Intuitively it is clear that the structure of the solution depends on the ratio of $m$ and the dynamical scale $\Lambda$ generated in this theory. As we will see below, there are two distinct cases - the Higgs and the Coulomb/confining phases - in this theory at large and small $\left|m_{0} / \Lambda\right|$, respectively.

In the Higgs phase the field $n^{i_{0}}$ develops a VEV. One can always choose $i_{0}=1$ and denote $n^{i_{0}}=n^{1} \equiv n$. There are $N$ equivalent choices, $N$ vacua. This corresponds to the spontaneous breaking of $Z_{N}$. Setting the background $A_{\alpha}$ field to zero, as in Sec. 5.1, and integrating out all $n_{i}$ except $n^{i_{0}}=n$ we arrive at

$$
\begin{aligned}
\mathcal{L}_{\mathrm{eff}} & =\left|\partial_{\alpha} n\right|^{2}+\left(D+\left|\sigma-m_{1}\right|^{2}\right)|n|^{2} \\
& +\frac{1}{4 \pi} \sum_{i=2}^{N}\left(D+\left|\sigma-m_{i}\right|^{2}\right)\left[1-\log \frac{D+\left|\sigma-m_{i}\right|^{2}}{\Lambda^{2}}\right] \\
& +\frac{1}{4 \pi} c \sum_{i=2}^{N}\left|\sigma-m_{i}\right|^{2}
\end{aligned}
$$

where

$$
c=\frac{1}{N} \sum_{i=2}^{N}\left(1-\frac{m_{i}}{m_{1}}\right) \log \frac{\left|m_{i}-m_{1}\right|^{2}}{\Lambda^{2}}
$$


and we used the renormalization condition

$$
\frac{2}{g_{0}^{2}}=\frac{N}{4 \pi} \ln \frac{M_{\mathrm{uv}}^{2}}{\Lambda^{2}} .
$$

This condition introduces the dynamical scale $\Lambda$ through dimensional transmutation, just like in Sec. 5.1.

Minimizing this effective potential with respect to $D, n$ and $\sigma$ we determine the vacuum values of these parameters. It is not surprising that $n_{\text {vac }}$ turns out to be exactly as it follows from the renormalized constraint $\left|n^{i}\right|^{2}=2 \beta$ in the Higgs phase,

$$
n_{\mathrm{vac}}=\left(\frac{N}{2 \pi} \log \left|\frac{m_{0}}{\Lambda}\right|\right)^{1 / 2}
$$

while

$$
D_{\mathrm{vac}}=0, \quad \sigma_{\mathrm{vac}}=m_{1} .
$$

It is also obvious that there are $N$ vacua corresponding to cyclic permutation. In each of them the $Z_{N}$ symmetry is spontaneously broken.

Substituting (68) and (69) in (65) we obtain the vacuum energy density,

$$
E_{\text {Higgs vac }}=\frac{N}{2 \pi} m_{0}^{2},
$$

where the parameter $m_{0}$ is assumed to be real and positive (see the bold line in Fig. 5). This formula is valid at

$$
m_{0} \geq \Lambda
$$

The Higgs phase has a clear-cut meaning at large $m_{0}$. The above result is compatible with intuition. We will see momentarily that the lower bound of the allowed domain, $m_{0}=\Lambda$, is the phase transition point (presumably, the phase transition is of the second order).

Now let us discuss the Coulomb/confining phase. At small $\left|m_{0}\right|$

$$
\sigma_{\mathrm{vac}}=0, \quad\left(n^{i}\right)_{\mathrm{vac}}=0 \text { for all } i, \quad i=1,2, \ldots, N,
$$

and

$$
D_{\mathrm{vac}}=\Lambda^{2}-m_{0}^{2} \text {. }
$$

The $Z_{N}$ symmetry remains unbroken. Hence, we deal with a unique vacuum. Inspecting Eq. (64) we conclude that in the saddle point the mass of all $n^{i}$ 


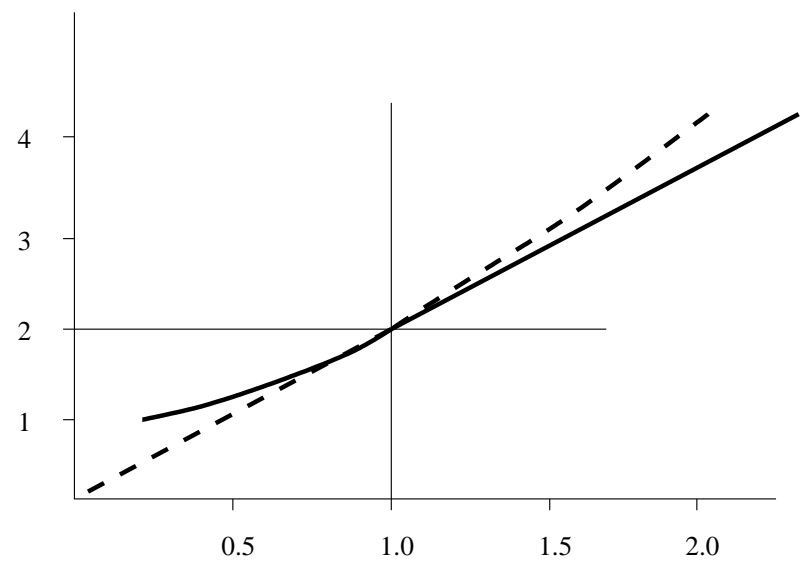

Figure 5: Normalized vacuum energies $\left(4 \pi E_{\mathrm{vac}} / N \Lambda^{2}\right)$ versus $m_{0}^{2} / \Lambda^{2}$. The solid line shows the actual vacuum energy, while dashed lines correspond to a formal extrapolation of the Higgs and Coulomb/confinement vacuum energies to unphysical values of $m$ below and above the phase transition point, respectively.

quanta is $\Lambda$, independent of the value of the mass deformation parameter $m_{0}$.

The vacuum energy in the Coulomb phase is obtained by substituting the vacuum values (72) and (73) in (65) and using expression (66) for the value of the constant $c$. In this way one arrives at

$$
E_{\text {Coulomb vac }}=\frac{N}{4 \pi}\left\{\Lambda^{2}+m_{0}^{2}+m_{0}^{2} \log \frac{m_{0}^{2}}{\Lambda^{2}}\right\},
$$

(see the solid line in Fig. 5).

At the point of the phase transition at $m_{0}=\Lambda$ the energy densities in the both phases coincide. Moreover, their first derivatives with respect to $m_{0}^{2}$ at this point coincide too. The dashed line corresponds to a formal extrapolation of the Higgs and Coulomb/confinement vacuum energies to "forbidden" values of $m_{0}^{2}$ below and above the phase transition point.

To reiterate, at $m_{0} \geq \Lambda$, at weak coupling, we have $N$ strictly degenerate vacua; the $Z_{N}$ symmetry is broken. At $m_{0} \leq \Lambda$ the $Z_{N}$ symmetry is unbroken, and the vacuum is unique. The order parameter which marks these vacua is the VEV of $n^{i}$.

Introduction of an additional axion field in this model is discussed in [43]. 


\subsection{Supersymmetric $\mathrm{CP}(N-1)$ with $\mathcal{N}=(2,2)$}

Two-dimensional $\mathrm{CP}(N-1)$ models with twisted masses and with $\mathcal{N}=(2,2)$ supersymmetry [44] emerge as effective low-energy theories on the world sheet of non-Abelian strings in a class of four-dimensional $\mathcal{N}=2$ gauge theories with unequal (s)quark masses [9], for a complete derivation see [18]. In the gauged formulation the $\operatorname{CP}(N-1)$ Lagrangian with the twisted masses (replacing the zero mass limit (25)) is (see e.g. [18, 27])

$$
\begin{aligned}
\mathcal{L} & =\left[|\mathcal{D} n|^{2}+2\left|\sigma-\frac{m^{l}}{\sqrt{2}}\right|^{2}\left|n^{l}\right|^{2}+i D\left(\left|n^{l}\right|^{2}-2 \beta\right)\right. \\
& +\bar{\xi}_{R} i \mathcal{D}_{L} \xi_{R}+\bar{\xi}_{L} i \mathcal{D}_{R} \xi_{L}+i \sqrt{2}\left(\sigma-\frac{m^{l}}{\sqrt{2}}\right) \bar{\xi}_{R l} \xi_{L}^{l}+i \sqrt{2}\left(\bar{\sigma}-\frac{\bar{m}^{l}}{\sqrt{2}}\right) \bar{\xi}_{L l} \xi_{R}^{l} \\
& \left.+\left(i \sqrt{2} \bar{\xi}_{R} \lambda_{L} n-i \sqrt{2} \bar{n} \lambda_{R} \xi_{L}+\text { H.c. }\right)\right]
\end{aligned}
$$

To solve the model in the large- $N$ limit one can basically repeat the derivation of Sec. 5.2 since the fields $n$ and $\xi$ enter in the Lagrangian bilinearly. Integrating them out yields

$$
\frac{\prod_{i=2}^{N} \operatorname{det}\left(-\partial_{k}^{2}+\left|\sqrt{2} \sigma-m_{i}\right|^{2}\right)}{\prod_{i=2}^{N} \operatorname{det}\left(-\partial_{k}^{2}+i D+\left|\sqrt{2} \sigma-m_{i}\right|^{2}\right)}
$$

and we obtain [27] an analog of the nonsupersymmetric formula (65) for the vacuum structure. Above we integrated over $N-1$ fields $n^{i}$ and $\xi^{i}$ with $i>1$. The resulting effective action is to be considered as a functional of $n^{1} \equiv n$, $D$ and $\sigma$. We will again assume that the twisted masses are $Z_{N}$ symmetric, see (63).

The ensuing effective Lagrangian is

$$
\begin{aligned}
\mathcal{L} & =\sum_{i=2}^{N} \frac{1}{4 \pi}\left\{\left(i D+\left|\sqrt{2} \sigma-m_{i}\right|^{2}\right)\left(\log \frac{M_{\mathrm{uv}}^{2}}{i D+\left|\sqrt{2} \sigma-m_{i}\right|^{2}}+1\right)\right. \\
& \left.-\left|\sqrt{2} \sigma-m_{i}\right|^{2}\left(\log \frac{M_{\mathrm{uv}}^{2}}{\left|\sqrt{2} \sigma-m_{i}\right|^{2}}+1\right)\right\},
\end{aligned}
$$


Using (67) for the bare coupling constant we can eliminate $M_{\mathrm{uv}}$ in a usual way. Then the effective potential as a function of $n, D$ and $\sigma$ fields takes the form

$$
\begin{aligned}
V_{\mathrm{eff}} & =\left(i D+\left|\sqrt{2} \sigma-m_{1}\right|^{2}\right)|n|^{2} \\
& -\frac{1}{4 \pi} \sum_{i=2}^{N}\left(i D+\left|\sqrt{2} \sigma-m_{i}\right|^{2}\right) \log \frac{i D+\left|\sqrt{2} \sigma-m_{i}\right|^{2}}{\Lambda^{2}} \\
& +\frac{1}{4 \pi} \sum_{i=2}^{N}\left|\sqrt{2} \sigma-m_{i}\right|^{2} \log \frac{\left|\sqrt{2} \sigma-m_{i}\right|^{2}}{\Lambda^{2}}+\frac{1}{4 \pi} i D(N-1) .
\end{aligned}
$$

Minimization of (78) gives two solutions: either

$$
i D+\left|\sqrt{2} \sigma-m_{1}\right|^{2}=0
$$

or

$$
n=0 \text {. }
$$

These two distinct solutions correspond to the weak and strong-coupling regimes of the theory, respectively. They are analogous to two phases we observed in Sec. 7.1. In the case at hand supersymmetry is unbroken but in both weak and strong-coupling regimes the $Z_{N}$ symmetry is spontaneously broken, and there are $N$ distinct vacua. At strong coupling, in the regime $n_{\mathrm{vac}}=0$, the order parameter is $\bar{\xi}_{R} \xi_{L}$ and its Hermitean conjugate.

As usual, supersymmetry suppresses phase transitions. The passage from weak (large $\left.\left|m_{0}\right|\right)$ and strong (small $\left|m_{0}\right|$ ) regimes presents a crossover rather than a phase transition. Supersymmetry is preserved in both regimes.

At large $\left|m_{0}\right|$

$$
D=0, \quad \sqrt{2} \sigma_{\text {vac }}=m_{1}, \quad\left|n_{\text {vac }}\right|^{2}=\frac{N}{2 \pi} \log \frac{m}{\Lambda} .
$$

This is similar to the Higgs phase in Sec. 7.1.

For small $\left|m_{0}\right|$ we have

$$
D=0, \quad n_{\mathrm{vac}}=0,
$$


while the vacuum equation on $\sigma$ can be written as

$$
\prod_{i=1}^{N}\left|\sqrt{2} \sigma-m_{i}\right|=\Lambda^{N}
$$

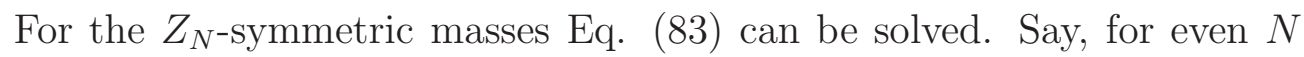
one can rewrite this equation in the form

$$
\left|(\sqrt{2} \sigma)^{N}-m^{N}\right|=\Lambda^{N}
$$

due to the fact that with the masses given in (63)

$$
\begin{aligned}
\sum m_{i} & =0, \\
\sum_{i, j ; i \neq j} m_{i} m_{j} & =0, \\
& \cdots \\
\sum_{i_{1}, i_{2}, \ldots, i_{N-1}} m_{i_{1}} m_{i_{2}} \ldots m_{i_{N-1}} & =0, \quad\left(i_{1} \neq i_{2} \neq \ldots \neq i_{N-1}\right) .
\end{aligned}
$$

Equation (84) has $N$ solutions (i.e. $N$ distinct vacua),

$$
\sqrt{2} \sigma=\left(\Lambda^{N}+m_{0}^{N}\right)^{1 / N} \exp \left(\frac{2 \pi i k}{N}\right), \quad k=1, \ldots, N
$$

The crossover occurs at $m_{0}=\Lambda$. The width of the crossover domain is not seen in the leading order in $1 / N$. In fact, it is exponentially small in $N$. The transition from weak to strong coupling is depicted in Fig. 6.

Summarizing, in both regimes - weak and strong coupling - supersymmetry is unbroken and there is no confinement of charged particles due to the fact that the photon (which becomes dynamical) acquires a mass. The spontaneous breaking of $Z_{N}$ implies $N$ degenerate vacua.

\subsection{Curves of marginal stability in (2.2) $\operatorname{CP}(N-1)$ with $Z_{N}$ twisted masses}

An exact twisted superpotential of the Veneziano-Yankielowicz type [45] is known to describe the $\mathcal{N}=(2,2)$ supersymmetric $\mathrm{CP}(N-1)$ model [46, 47, 


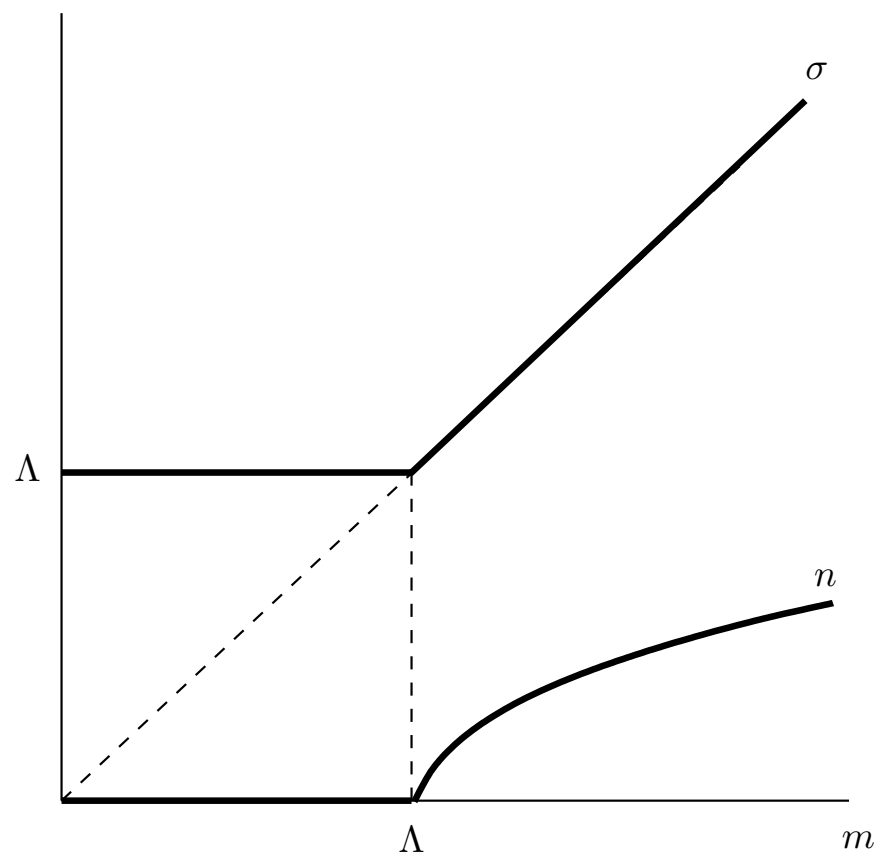

Figure 6: Plots of $n$ and $\sigma$ VEVs (thick lines) vs. $m_{0}$ in the $\mathcal{N}=(2,2) \operatorname{CP}(N-1)$ model with the $Z_{N}$-symmetric twisted masses. 
36, 44, 48]. Integrating out the fields $n^{P}$ and $\rho^{K}$ we obtain the following exact twisted superpotential:

$$
\mathcal{W}_{\mathrm{CP}(N-1)}(\sigma)=\frac{1}{4 \pi}\left\{\sum_{l=1}^{N}\left(\sqrt{2} \sigma-m_{l}\right) \ln \frac{\sqrt{2} \sigma-m_{l}}{\Lambda}-N \sqrt{2} \sigma\right\},
$$

where we use one and the same notation $\sigma$ for the twisted superfield [36] and its lowest scalar component. Minimizing this superpotential with respect to $\sigma$ we get the equation for the $\sigma$-field $\mathrm{VEVs}$,

$$
\prod_{l=1}^{N}\left(\sqrt{2} \sigma-m_{l}\right)=\Lambda^{N}
$$

This equation has $N$ roots $\sigma_{p}(p=1, \ldots, N)$ associated with $N$ vacua of the $\mathrm{CP}(N-1)$ model. Note that this exact equation is a holomorphic version of Eq. (83) which appears in the large- $N$ solution. It takes into account that chiral $\mathrm{U}(1)_{R}$ symmetry is broken by chiral anomaly down to discrete $Z_{2 N}$ symmetry. This is the reason for the presence of $N$ distinct vacua.

The masses of the BPS kinks interpolating between the vacua $\sigma_{p}$ and $\sigma_{p^{\prime}}$ are given by the appropriate differences of the superpotential (87) calculated at distinct roots [44, 48, 49],

$$
M_{p p^{\prime}}^{\mathrm{BPS}}=2\left|\mathcal{W}_{\mathrm{CP}(N-1)}\left(\sigma_{p^{\prime}}\right)-\mathcal{W}_{\mathrm{CP}(N-1)}\left(\sigma_{p}\right)\right|, \quad p, p^{\prime}=1, \ldots, N
$$

In addition to kinks, the BPS spectrum of the model contains elementary excitations with masses given by $\left|m_{l}-m_{p}\right|(l=1, \ldots, N$ and $p=1, \ldots, N)$.

Due to the presence of branches in the logarithmic functions in (87) each kink comes together with a tower of dyonic kinks carrying global U(1) charges. The dyonic kinks are reflected in (89) through terms

$$
\text { integer } \times i m_{l}
$$

with different $l$ which appear from the logarithm branches. We stress that all these kinks with the imaginary part (90) in the mass formula (89) interpolate between the same pair of vacua: $p$ and $p^{\prime}$.

Generically there are way too many choices in (89). Not all of them are realized. Moreover, the kinks present in the quasiclassical domain (i.e. at large $\left.\left|m_{0}\right|\right)$ decay on the curves of marginal stability (CMS) or form new bound states. Therefore, the quasiclassical spectrum outside CMS and the quantum 
spectrum inside CMS (i.e. at small $\left|m_{0}\right|$ ) are different. This phenomenon is referred to as "wall crossing." There exists a general procedure [50] which allows one to determine the full BPS spectrum starting from the strong coupling spectrum inside CMS. However, this procedure is rather cumbersome. In certain cases one can use a simpler approach based on analysis of various limits. Below we will briefly review the BPS spectra and CMS in $\mathrm{CP}(1)$ and $\mathrm{CP}(2)$ with the $Z_{N}$ twisted masses [48, 51, 52].

These CMS were obtained by matching the weak coupling BPS spectrum found using semiclassical considerations with the strong coupling spectrum found from mirror representation of the $\mathrm{CP}(N-1)$ model [53]. The later spectrum includes only $N$ kinks which become massless at strong coupling.

The strong coupling spectrum of the $\mathrm{CP}(1)$ model includes two BPS states with the following masses:

$$
M_{0}=\left|m_{D}^{\mathrm{CP}(1)}+i m_{1}\right|, \quad M_{1}=\left|m_{D}^{\mathrm{CP}(1)}+i m_{2}\right|,
$$

where

$$
m_{D}^{\mathrm{CP}(1)}=\frac{1}{\pi}\left[2 \sqrt{m_{0}^{2}+\Lambda^{2}}-m_{0} \log \frac{\sqrt{m_{0}^{2}+\Lambda^{2}}+m_{0}}{\sqrt{m_{0}^{2}+\Lambda^{2}}-m_{0}}\right],
$$

while $m_{1}, m_{2}$ are given in (63) with $N=2$, namely, $m_{1}=-m_{0}, m_{2}=m_{0}$.

The weak coupling spectrum of the $\mathrm{CP}(1)$ model includes the tower of dyonic kinks

$$
M_{n}=\left|m_{D}^{\mathrm{CP}(1)}+i m_{1}+i n\left(m_{2}-m_{1}\right)\right|,
$$

where $n$ is an integer. The two states of the strong coupling spectrum (91) belong to this tower with $n=0,1$. Other states from this tower as well as elementary (i.e. non-kink) states decay on the closed single CMS around the origin in the $m_{0}^{2}$ complex plane [51].

Now let us briefly discuss a more contrived situation, the $\mathrm{CP}(2)$ model with the $Z_{3}$ twisted masses. For simplicity we will restrict ourselves to kinks interpolating between the third and first vacua $\left(\sqrt{2} \sigma_{3} \approx m_{3}\right.$ and $\sqrt{2} \sigma_{1} \approx m_{1}$ in the large mass limit). The strong coupling spectrum of the $\mathrm{CP}(2)$ model consists of three states

$$
M_{k}^{13}=\left|m_{D}^{\mathrm{CP}(2)}+i m_{k}\right|, \quad k=1,2,3,
$$


where

$$
m_{D}^{\mathrm{CP}(2)}=-\frac{1}{2 \pi}\left(e^{2 \pi i / 3}-1\right)\left\{3 \sqrt[3]{m_{0}^{3}+\Lambda^{3}}+\sum_{j} m_{j} \log \frac{\sqrt[3]{m_{0}^{3}+\Lambda^{3}}-m_{j}}{\Lambda}\right\}
$$

and the mass terms $m_{k}$ are given in (63) with $N=3$.

The weak coupling spectrum of the $\mathrm{CP}(2)$ model includes two towers of dyonic kinks

$$
\begin{aligned}
& M_{n_{1}}^{13}=\left|m_{D}^{\mathrm{CP}(2)}+i m_{3}+i n_{1}\left(m_{1}-m_{3}\right)\right| \text { and } \\
& M_{n_{2}}^{13}=\left|m_{D}^{\mathrm{CP}(2)}+i m_{3}+i n_{2}\left(m_{1}-m_{3}\right)+i\left(m_{3}-m_{2}\right)\right|,
\end{aligned}
$$

plus elementary states. Here $n_{1}$ and $n_{2}$ are integers. The two states of the strong coupling spectrum (94) with $k=3,1$ both belong to the first tower. The $\mathrm{CP}(2)$ model with the $Z_{3}$ masses has several CMS where all other states except these two decay [52], see Fig 7 . The third kink of the strong coupling spectrum (with $k=2$ ) does not make it to the weak coupling domain. It decays on the most inner curve in Fig. 7.

\section{$8 \quad$ Weighted $\operatorname{CP}(N, M)$ models and $z n$ model}

Considering $\mathcal{N}=2$ bulk theories of the type discussed in Sec. 2 with $N_{f}>N$ (here $N_{f}$ is the number of flavors) we arrive at the so-called semilocal nonAbelian strings [54, 55, 56, 57]. Instead of $N_{f}$ we can introduce a positive number $M$,

$$
N_{f}=N+M \text {. }
$$

The semilocal string solutions on the Higgs branches (typical for multiflavor theories) usually are not fixed-radius strings, but, rather, possess radial moduli $\rho^{k}$, also known as the size moduli (see [58] for a review of the Abelian semilocal strings).

As previously, the orientational moduli of the semilocal non-Abelian string can be described by a complex vector $n^{P}$ (here $\left.P=1, \ldots, N\right)$, while its size moduli are parametrized by a complex vector $\rho^{K}$ (here $\left.K=N+1, \ldots, N+M\right)$.

Originally it was conjectured [54] (on the basis of string theory arguments) that the effective two-dimensional sigma model describing low-energy dynamics on the semilocal string is the so-called weighted $\mathrm{CP}(N, M)$ model. This 


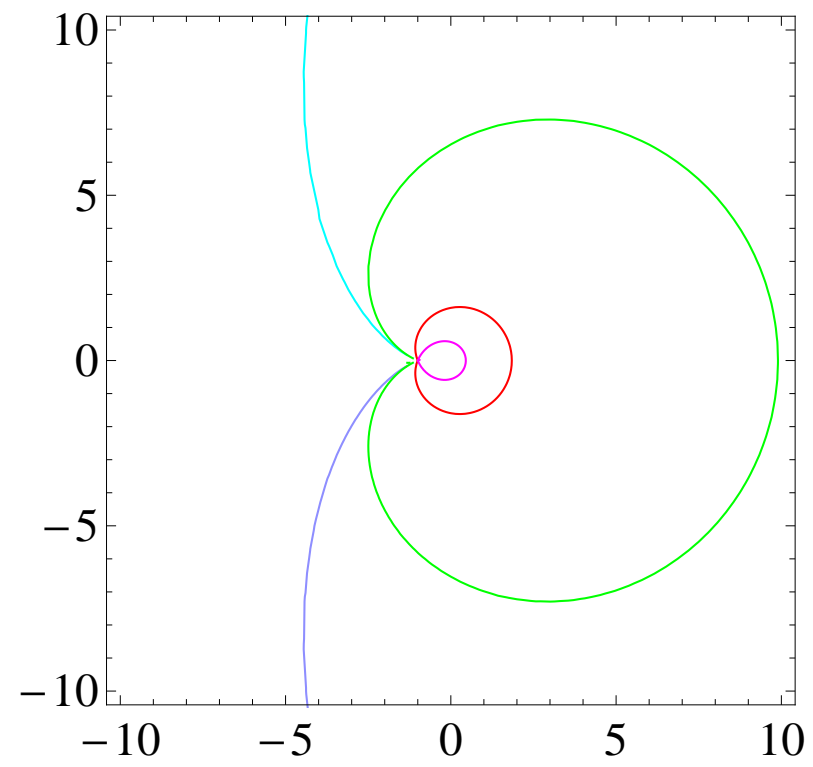

Figure 7: The decay curves of $\mathrm{CP}^{2}$ in $m_{0}^{3}$ plane.

turned out to be not quite correct. The world-sheet theory of the moduli fields was derived in [57, 59] and is known as the $z n$ model. Its Lagrangian is

$$
\begin{aligned}
\mathcal{L}_{z n} & =\left|\partial_{\alpha}\left(n^{P} \rho^{K}\right)\right|^{2}+\left|\mathcal{D}_{\alpha} n^{P}\right|^{2}+\left|m_{K}-m_{P}\right|^{2}\left|n^{P}\right|^{2}\left|\rho^{K}\right|^{2} \\
& +\left|\sqrt{2} \sigma-m_{P}\right|^{2}\left|n^{P}\right|^{2}+i D\left(\left|n^{P}\right|^{2}-2 \beta\right)^{2}, \\
P & =1, \ldots, N, \quad K=N+1, \ldots, N+M,
\end{aligned}
$$

The $z n$ model so far remains largely unexplored. We refer the reader to the original papers for a brief discussion.

The $z n$ model is similar but not identical to the weighted $\mathrm{CP}(N, M)$ model. However, it was demonstrated [57, 59] that its vacuum structure and BPS spectrum coincide with those of the $\mathcal{N}=2$ weighted $\operatorname{CP}(N, M)$ model. Moreover, at $N \rightarrow \infty$ the $z n$ model and the weighted $\operatorname{CP}(N, M)$ model coincide. Thus, the difference between them lies in the non-BPS sector at finite $N$. 
Technically, it seems more convenient to work with the weighted $\operatorname{CP}(N, M)$ model. The bosonic part of its Lagrangian is

$$
\begin{aligned}
\mathcal{L}_{\mathrm{WCP}} & =\left|\mathcal{D}_{\alpha} n^{P}\right|^{2}+\left|\tilde{\mathcal{D}}_{\alpha} \rho^{K}\right|^{2}+\left|\sqrt{2} \sigma-m_{P}\right|^{2}\left|n^{P}\right|^{2} \\
& +\left|\sqrt{2} \sigma-m_{K}\right|^{2}\left|\rho^{K}\right|^{2}+i D\left(\left|n^{P}\right|^{2}-\left|\rho^{K}\right|^{2}-2 \beta\right)^{2} \\
P & =1, \ldots, N, \quad K=N+1, \ldots, N+M,
\end{aligned}
$$

where

$$
\mathcal{D}_{\alpha}=\partial_{\alpha}-i A_{\alpha}, \quad \tilde{\mathcal{D}}_{\alpha}=\partial_{\alpha}=i A_{\alpha} .
$$

The mass terms $M_{K}$ and $M_{P}$ in (99) are viewed as generic in this section.

The fields $n^{P}$ and $\rho^{K}$ have the opposite charges, +1 and -1 , with respect to the auxiliary $\mathrm{U}(1)$ gauge field. This seemingly insignificant detail is crucial. Strictly speaking, the name 'weighted $\mathrm{CP}$ ' model is misleading since the geometry of the target-space following from (99) has nothing to do with the $\mathrm{CP}(N-1)$ geometry in which all target space covariant quantities reduce to the metric, see (18). The weighted $\mathrm{CP}$ models are not even renormalizable in the usual sense of this word. Nevertheless, the large- $N$ solution exists and is unique [60]. We will discuss it in more detail in Sec. 9.6.

\section{$9 \quad$ Heterotic models}

Heterotic two-dimensional models we will discuss below have two chiral supercharges, say, $Q_{L}$ and $\bar{Q}_{L}$ with the defining anticommutator

$$
\left\{Q_{L}, \bar{Q}_{L}\right\}=2(H-P) \text {. }
$$

They are known as $\mathcal{N}=(0,2)$ supersymmetric sigma models 4 Previously they were studied mainly from the mathematical perspective $20,21,23,24$. They can be divided into two classes: the so-called minimal and nonminimal models. This classification is in a sense similar to pure Yang-Mills theories and Yang-Mills theories with matter. Later we will explain the difference between these two classes in more detail. In particular, the minimal $\mathrm{CP}(1)$ model was considered in [22]. This minimal model cannot be extended to

\footnotetext{
${ }^{4}$ In Sec. 9.6 we will briefly comment on a $\mathcal{N}=(0,1)$ model.
} 
$\mathrm{CP}(N-1)$ with $N>2$. The general hypercurrent structure in $\mathcal{N}=(0,2)$ was analyzed in [25]. In what follows we will focus on those heterotic twodimensional models that are obtained on the world sheet of non-Abelian strings.

\subsection{How heterotic models appear}

If the bulk four-dimensional theory has $\mathcal{N}=2$ and supports 1/2-BPS strings, then the low-energy theory on its world sheet has four supercharges and, thus, possesses $\mathcal{N}=(2,2)$ supersymmetry. Now, if we slightly deform the bulk theory breaking $\mathcal{N}=2$ down to $\mathcal{N}=1$ we will have four supercharges in the bulk. For small deformations BPS saturation remains intact and so does the the target space of the two-dimensional sigma model. Now, the world-sheet model must have two, not four supercharges. However, Zumino's theorem tells us that given a Kähler target space any supersymmetric nonchiral model is automatically uplifted to $\mathcal{N}=(2,2)$, i.e. four supercharges.

A way out was suggested by Edalati and Tong [16] who conjectured a nonminimal $\mathcal{N}=(0,2)$ model on the string world sheet in the case of nonvanishing $\mathcal{N}=2$ breaking deformation in the bulk (see also [61]). This nonminimal theory was derived by Shifman and Yung [17] from the analysis of the string solution. The nonminimal theory, as it emerged on the string world sheet, has no twisted masses. In fact, even today we do not know which bulk theory might result in the nonminimal heterotic model with twisted masses. However, the inclusion of the twisted masses is straightforward in the twodimensional model per se, without any reference to the bulk theory. This is the model to be discussed below too.

Large- $N$ solutions of the heterotic models generically exhibit spontaneous breaking of supersymmetry. For nonvanishing twisted masses this breaking occurs at the tree level. 


\subsection{Minimal vs. nonminimal $\mathrm{CP}(N-1)$ models with $\mathcal{N}=(0,2)$ supersymmetry}

\subsubsection{Geometric formulation}

The minimal model can be obtained from (12) by keeping only left-handed fermions and discarding all right-handed fermions,

$$
\mathcal{L}_{\mathcal{N}=(0,2)}=G_{i \bar{j}}\left[\partial^{\mu} \phi^{\dagger} \bar{j} \partial_{\mu} \phi^{i}+i \bar{\psi}_{L}^{\bar{j}} \mathcal{D}_{R} \psi_{L}^{i}\right],
$$

where

$$
\mathcal{D}_{R} \psi_{L}^{i}=\partial_{R} \psi_{L}^{i}+\Gamma_{k l}^{i}\left(\partial_{R} \phi^{k}\right) \psi_{L}^{l},
$$

and

$$
\partial_{R} \equiv \partial_{t}-\partial_{z}
$$

The fields $\phi$ and $\psi_{L}$ form an $(0,2)$ supermultiplet. In terms of $\mathcal{N}=(0,2)$ superfields [20] one can act as follows. Introduce a superfield

$$
A=\phi\left(x_{R}+2 i \theta^{\dagger} \theta, x_{L}\right)+\sqrt{2} \theta \psi_{L}\left(x_{R}+2 i \theta^{\dagger} \theta, x_{L}\right),
$$

where $\theta$ is a single (right-handed) complex Grassmann variable on the $(0,2)$ superspace, and

$$
x_{L}=t-z \equiv x^{0}-x^{1}, \quad x_{R}=t+z \equiv x^{0}+x^{1} .
$$

Then

$$
\begin{aligned}
\mathcal{L}_{\text {min }} & =\frac{1}{2} \int d \theta d \bar{\theta}\left[K_{i}\left(A, A^{\dagger}\right) i \partial_{R} A^{i}+\text { H.c. }\right] \\
& =-\frac{1}{4} \int d \theta G_{i \bar{j}}\left(A, A^{\dagger}\right)\left(\bar{D} A^{\dagger \bar{j}}\right) i \partial_{R} A^{i}+\text { H.c. }
\end{aligned}
$$

Warning: Due to an anomaly pointed out in [40] the heterotic minimal model is self-consistent only for $C P(1)$ (see also [31]). Minimal $C P(N-1)$ models with $N>2$ do not exist. However, minimal heterotic $O(N)$ models exist for any $N$. For $N>3$ they have $(0,1)$ supersymmetry. For $N=3$ we have $O(3)=C P(1)$. Nonminimal models presented in (109) exist for $C P(N-1)$ at any $N$.

Alternatively, one can start from Eq. (25) and discard all terms containing $\xi_{R}$. 
One last remark is in order here concerning the minimal $\mathrm{CP}(1)$ model presented in (107). This is a strongly coupled theory. Since large- $N$ expansion is unavailable, we cannot solve it by virtue of the large- $N$ expansion (we will apply it, however, to nonminimal heterotic $\mathrm{CP}(N-1))$. Nevertheless, one feature of this model is known. As was shown in [25], current algebra in this model allows for a nonperturbative Schwinger term (see Eq. (5.7) in [25]), namely, $C \sim \Lambda^{2} \sim M_{\mathrm{uv}}^{2} \exp \left(-\frac{4 \pi}{g_{0}^{2}}\right)$. This Schwinger term is saturated by a single instanton due to the fact that in the model at hand it has just two fermion zero modes. The occurrence of this Swinger term implies spontaneous supersymmetry breaking. The interpolating field for Goldstino is

$$
g \sim R_{i \bar{j}}\left(\partial_{R} \phi^{i}\right) \bar{\psi}_{L}^{\bar{j}}
$$

Spontaneous breaking of supersymmetry will be explicit in the large- $N$ solution of the nonminimal heterotic $\mathrm{CP}(N-1)$.

The bulk theories supporting non-Abelian strings are usually obtained by deforming $\mathcal{N}=2$ theories by a mass term of the adjoint superfield which breaks bulk supersymmetry down to $\mathcal{N}=1$. In this case the moduli fields on the string include all those inherent to the $\mathcal{N}=(2,2) \operatorname{CP}(N-1)$ model plus an extra $\mathcal{N}=(0,2)$ supermultiplet with a peculiar interaction. The heterotic model obtained in this way is to be referred to as nonminimal. In the geometric formulation its Lagrangian is

$$
\begin{aligned}
\mathcal{L} & =G_{i \bar{j}}\left[\partial_{R} \phi^{\dagger \bar{j}} \partial_{L} \phi^{i}+\psi_{L}^{\dagger \bar{j}} i \mathcal{D}_{R} \psi_{L}^{i}+Z \psi_{R}^{\dagger \bar{j}} i \mathcal{D}_{L} \psi_{R}^{i}\right]+Z R_{i \bar{j} k \bar{l}} \psi_{L}^{\dagger \bar{j}} \psi_{L}^{i} \psi_{R}^{\dagger \bar{l}} \psi_{R}^{k} \\
& +\mathcal{Z} \zeta_{R}^{\dagger} i \partial_{L} \zeta_{R}+\left[\kappa \zeta_{R} G_{i \bar{j}}\left(i \partial_{L} \phi^{\dagger \bar{j}}\right) \psi_{R}^{i}+\text { H.c. }\right]+\frac{|\kappa|^{2}}{Z} \zeta_{R}^{\dagger} \zeta_{R}\left(G_{i \bar{j}} \psi_{L}^{\dagger \bar{j}} \psi_{L}^{i}\right) \\
& -\frac{|\kappa|^{2}}{\mathcal{Z}}\left(G_{i \bar{j}} \psi_{L}^{\dagger \bar{j}} \psi_{R}^{i}\right)\left(G_{k \bar{l}} \psi_{R}^{\dagger \bar{l}} \psi_{L}^{k}\right) .
\end{aligned}
$$

Here $\mathcal{D}_{L, R}$ are covariant derivatives,

$$
\mathcal{D}_{L, R} \psi_{R, L}^{i}=\partial_{L, R} \psi_{R, L}^{i}+\Gamma_{k l}^{i} \partial_{L, R} \phi^{k} \psi_{R, L}^{l}
$$

The first line in (109) coincides with the $(2,2)$ Lagrangian in Eq. (12). The second and third lines present a heterotic deformation. The right-handed fermion field $\zeta_{R}$ is absent in the $(2,2)$ model. 
In terms of superfields the nonminimal heterotic model can be written as follows:

$$
\begin{aligned}
\mathcal{L}= & -\frac{1}{2} \int d \theta\left[\frac{1}{2} G_{i \bar{j}}\left(A, A^{\dagger}\right)\left(\bar{D} A^{\dagger \bar{j}}\right) i \partial_{R} A^{i}-\kappa G_{i \bar{j}}\left(A, A^{\dagger}\right)\left(\bar{D} A^{\dagger \bar{j}}\right) \mathcal{B} B^{i}+\text { H.c. }\right] \\
& +\frac{1}{2} \int d^{2} \theta\left[Z G_{i \bar{j}}\left(A, A^{\dagger}\right) B^{\dagger \bar{j}} B^{i}+\mathcal{Z} \mathcal{B}^{\dagger} \mathcal{B}\right]
\end{aligned}
$$

where $\kappa$ is the deformation parameter, and the extra (compared to the minimal model) $(0,2)$ superfields are

$$
\begin{aligned}
& \mathcal{B}=\zeta_{R}\left(x_{R}+2 i \theta^{\dagger} \theta, x_{L}\right)+\sqrt{2} \theta F_{\zeta}\left(x_{R}+2 i \theta^{\dagger} \theta, x_{L}\right) \text { and } \\
& B=\psi_{R}\left(x_{R}+2 i \theta^{\dagger} \theta, x_{L}\right)+\sqrt{2} \theta F_{\psi}\left(x_{R}+2 i \theta^{\dagger} \theta, x_{L}\right) .
\end{aligned}
$$

On mass shell both $\mathcal{B}$ and $B$ contain one fermion degree of freedom, $\zeta_{R}$ and $\psi_{R}$, respectively.

\subsubsection{Gauged formulation}

The gauged formulation is most convenient for large- $N$ solution. Translation of the Lagrangian (109) in the gauged formulation yields the Lagrangian (A.6) presented in the Appendix. Technically, it is slightly more convenient to work with an equivalent Lagrangian

$$
\begin{aligned}
\mathcal{L}_{(0,2)} & =\bar{\zeta}_{R} i \partial_{L} \zeta_{R}+\left[2 i \omega \bar{\lambda}_{L} \zeta_{R}+\text { H.c. }\right] \\
& +\left|\mathcal{D}_{\mu} n^{l}\right|^{2}+2|\sigma|^{2}\left|n^{l}\right|^{2}+i D\left(\left|n^{l}\right|^{2}-2 \beta\right) \\
& +\bar{\xi}_{l R} i \mathcal{D}_{L} \xi_{R}^{l}+\bar{\xi}_{l L} i \mathcal{D}_{R} \xi_{L}^{l} \\
& +\left[i \sqrt{2} \sigma \bar{\xi}_{l R} \xi_{L}^{l}+i \sqrt{2} \bar{n}_{l}\left(\lambda_{R} \xi_{L}^{l}-\lambda_{L} \xi_{R}^{l}\right)+\text { H.c. }\right]+4|\omega|^{2}|\sigma|^{2}
\end{aligned}
$$

(equation (A.1) in the Appendix). The proof of equivalence is outlined in the Appendix. The deformation constant $\omega$ is related to $\kappa$ in (109) as follows:

$$
\begin{aligned}
\kappa \beta & =\frac{\omega}{\sqrt{2}}, \\
\mathcal{Z}_{0} & =1+\frac{|\omega|^{2}}{\beta}, \quad Z=1 .
\end{aligned}
$$


The deformation parameter $\omega$ is renormalization-group invariant, see Sec. 9.3. In the large- $N$ solution we will see that physical effects are determined by an $N$-independent deformation parameter,

$$
u=\frac{8 \pi}{N}|\omega|^{2}=\frac{16 \pi}{N g^{2}} \frac{\kappa^{2}}{g^{2}} .
$$

Both constants, $\kappa^{2}$ and $g^{2}$ scale with $N$ as $1 / N$.

\subsubsection{Twisted masses}

Twisted masses were added in [18, 27]. The corresponding expressions are quite bulky. The interested reader is referred to the original publications. A novel element worth noting is as follows. In the absence of the heterotic deformation the $\mathrm{CP}(N-1)$ model has $N-1$ complex twisted mass parameters, see Sec. 6. With $\kappa \neq 0$ the number of independent complex mass parameters generally speaking increases. In the generic case the nonminimal $(0,2)$ model will have $N$ independent mass parameters.

\subsection{Beta functions}

All models under consideration in this review paper are asymptotically free. As was noted by Polyakov in 1975 [33] at one loop only the bosonic fields contribute to the $\beta$ functions. Fermion contribution shows up at the two-loop level.

The exact all-loop $\beta$ function in the minimal $\mathrm{CP}(1)$ model was found in [30]. It has the form

$$
\beta_{g(0,2) \min }=-\frac{g^{4}}{2 \pi}\left(1-\frac{g^{2}}{4 \pi}\right)^{-1}
$$

where $g^{2}$ is the coupling constant in the Kähler potential and metric.

Its structure is perfectly analogous to that of the NSVZ $\beta$ function in four-dimensional $\mathcal{N}=1$ gluodynamics [62, 63]. In fact, the above twodimensional model is the closest analog of $\mathcal{N}=1$ four-dimensional theories one can think of. One can show [30] that the analogy extends further than Eq. (117) and is maintained when one introduces "matter" fields. Then the $\beta$ function (117) acquires a numerator typical of the NSVZ $\beta$ function in the presence of matter. 
In the nonminimal model one deals with two coupling constants, $g^{2}$ appearing in the metric, and the deformation parameter $\kappa$. At one loop the corresponding $\beta$ functions were calculated in [29], while the two-loop loop corrections and an exact relation between $\beta_{g}$ and the anomalous dimensions $\gamma$ were found in [31] (see also [29]),

$$
\beta_{g}=\mu \frac{d g^{2}}{d \mu}=-\frac{g^{2}}{4 \pi} \frac{T_{G} g^{2}\left(1+\gamma_{\psi_{R}} / 2\right)-h^{2}\left(\gamma_{\psi_{R}}+\gamma_{\zeta}\right)}{1-\left(h^{2} / 4 \pi\right)}
$$

where $\gamma_{\zeta}$ and $\gamma_{\psi_{R}}$ are the anomalous dimensions of the corresponding fields, which to the leading order are proportional to

$$
h^{2}=\frac{|\kappa|^{2}}{Z \mathcal{Z}},
$$

Here $Z$ and $\mathcal{Z}$ are filed renormalization constants for $\psi_{R}$ and $\zeta_{R}$ respectively (for their definition see [31]). At one loop [29]

$$
\gamma \equiv \gamma_{\psi_{R}}+\gamma_{\zeta}=\frac{N h^{2}}{2 \pi} .
$$

The two-loop anomalous dimensions (which are also known [31) give us explicit expression for $\beta_{g}$ at three loops.

One can view $h^{2}$ as the second coupling constant - the one responsible for the $\mathcal{N}=(2,2) \rightarrow(0,2)$ breaking. It is convenient to consider the ratio

$$
\rho \equiv h^{2} / g^{2} .
$$

the exact relation for the corresponding $\beta$ function is

$$
\beta_{\rho}=\rho\left[\frac{1}{g^{2}} \beta_{g}+\gamma\right] .
$$

An explicit expression for $\beta_{\rho}$ exists [28, 31] up to two loops,

$$
\beta_{\rho}^{(2)}=N \frac{g^{2}}{2 \pi} \frac{\rho}{1-\left(h^{2} / 4 \pi\right)}\left(\rho-\frac{1}{2}\right) .
$$

It has an infrared fixed pint at $\rho=1 / 2$ (see Fig. 8). Whether the fixed point at $\rho=1 / 2$ is an exact statement or it does not hold in the third and higher loops is not known. 


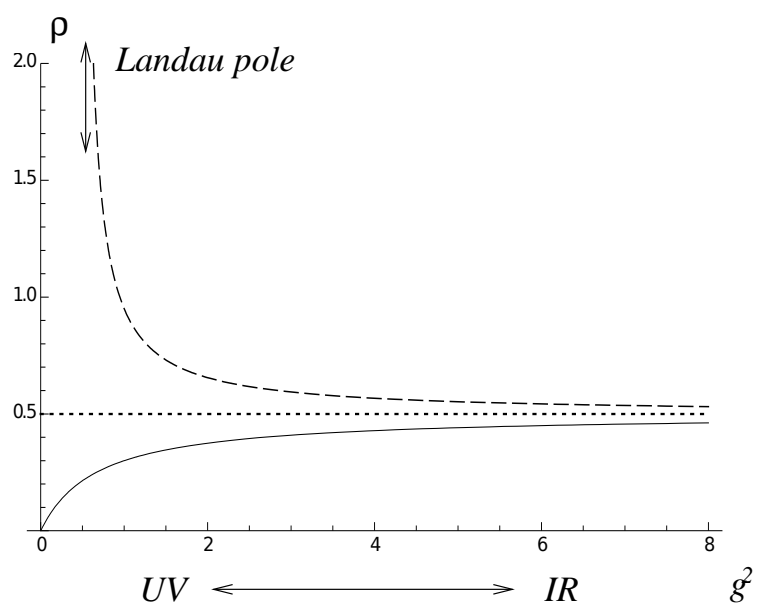

Figure 8: Infrared fixed point in $\beta_{\rho}$.

Another consequence from Eqs. (118) and (120) is as follows. In the limit $N \rightarrow \infty$ the constant $h^{2}$ scales as $1 / N$, implying that $\beta_{g}$ reduces to one loop and becomes exactly the same as in the undeformed $\mathcal{N}=(2,2)$ $\mathrm{CP}(N-1)$ model. This is in full agreement with the large- $N$ solution of the nonminimal heterotic model to be presented below. The combination $\kappa^{2} / g^{4}$ is renormalization-group invariant,

$$
\frac{\kappa^{2}}{g^{4}}=\mathrm{RGI},
$$

cf. Eqs. (114) and(116).

\subsection{Large- $N$ solution of nonminimal $\operatorname{CP}(N-1)$}

This model was solved with the $Z_{N}$ symmetric twisted masses [27] and arbitrary value of the mass parameter $m_{0}$. This solution includes of course the massless heterotic model [26] as a limiting case $m_{0}=0$. Therefore, we will pass directly to the nonminimal model with the $Z_{N}$ symmetric twisted masses.

One brief remark is in order before this passage. At small values of $u$, vanishing mass parameter $m_{0}$, and arbitrary (i.e. not necessarily large) $N$ it is easy to find both the Goldstino and the vacuum energy,

$$
g \sim \omega\left\langle R_{i \bar{j}} \bar{\psi}_{R}^{\bar{j}} \psi_{L}^{i}\right\rangle_{\text {vac }} \zeta_{R}
$$




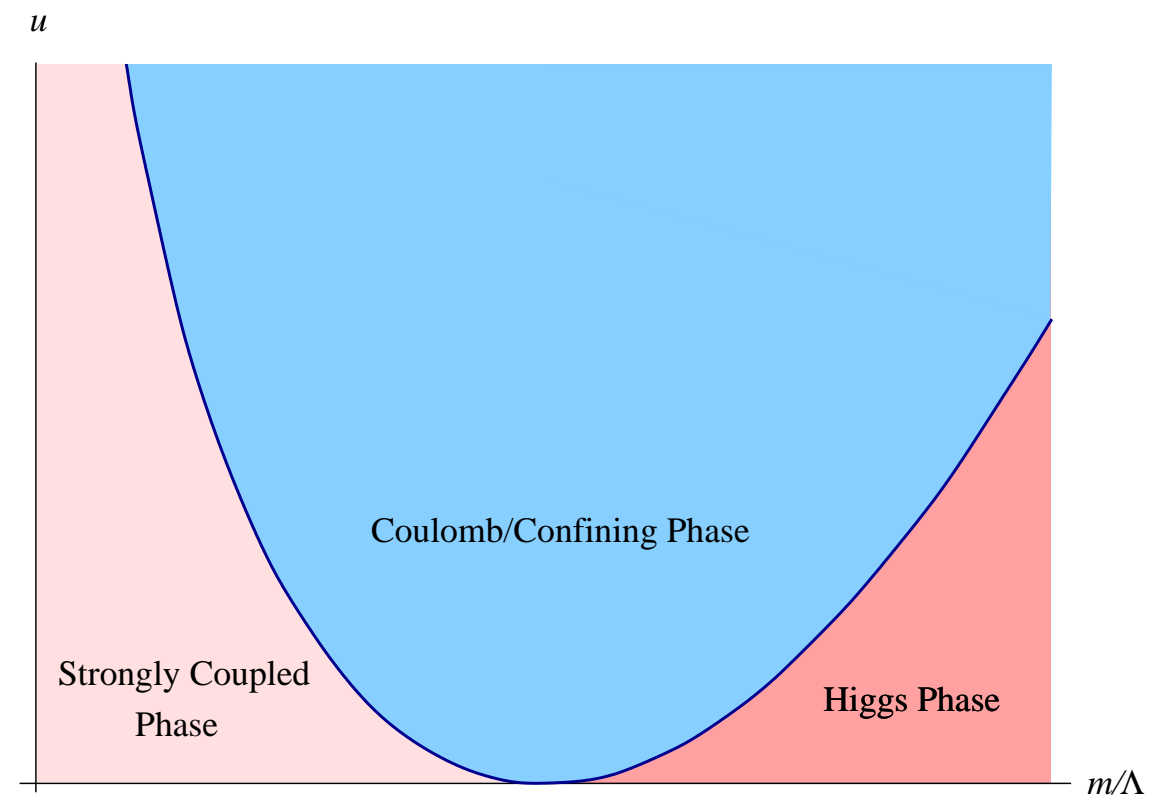

Figure 9: The phase diagram of the twisted-mass deformed heterotic $\operatorname{CP}(N-1)$ theory in the plane $u$ and $m_{0}$ where $m_{0}$ is assumed to be real. The parameter $u$ denotes the amount of deformation, $u=\frac{8 \pi}{N}|\omega|^{2}$.

where the vacuum averaging is performed in the undeformed $(2,2)$ massless $\mathrm{CP}(N-1)$ model, see Eqs. (6.26) and (6.27) in [17]. The extra right-handed field $\zeta_{R}$ plays the role of Goldstino.

Now, let us dicuss the solution found in [27]. Conceptually, the strategy of solving this model at large $N$ is similar to that described in Sec. 7. Since in the model at hand we have two parameters, $u$ and $m_{0}$, we discover a rather rich and not quite trivial phase diagram, in which we observe phases with broken or unbroken $Z_{N}$ symmetry. If $u \neq 0$ we have two phases with the broken $Z_{N}$ symmetry, on the left and on the right in Fig. 9. The first $Z_{N}$ phase is strongly coupled, the second (the Higgs phase) is weakly coupled. In the middle lies the phase of unbroken $Z_{N}$ symmetry, in which the vacuum is unique, the photon does not acquire a mass, and the corresponding dynamical regime is that of charge confinement.

Analytical solution for the vacuum structure is easier to obtain at large deformations, $u \gg 1$. 


\subsubsection{Strong coupling phase with broken $Z_{N}$}

This phase occurs at very small masses, namely,

$$
m_{0} \leq \Lambda e^{-u / 2}, \quad u \gg 1
$$

In this phase we have

$$
|n|=0, \quad i D \approx \Lambda^{2},
$$

while the vacuum value of the $\sigma$ field is

$$
\sqrt{2}\langle\sigma\rangle_{\mathrm{vac}}=e^{\frac{2 \pi i}{N} k} \Lambda e^{-u / 2}, \quad k=1, \ldots,(N) .
$$

The vacuum value of $\sigma$ is exponentially small at large $u$. The bound $m_{0}<$ $|\sqrt{2} \sigma|$ translates into the condition (126) for $m_{0}$. For simplicity we will assume in this section $m_{0}$ to be real and positive.

We have $N$ degenerate vacua in this phase. The chiral $Z_{2 N}$ symmetry is broken down to $Z_{2}$, the order parameter is $\langle\sigma\rangle$. Moreover, the absolute value of $\sigma$ in these vacua does not depend on $m$. This solution essentially coincides with one obtained in [26] in the massless case. In this aspect the situation

is quite similar to the strong coupling phase of the $\mathcal{N}=(2,2)$ model. The difference is that the absolute value of $\sigma$ depends now on $u$ and becomes exponentially small in the limit $u \gg 1$.

The vacuum energy is positive (see Fig. 10). Supersymmetry is spontaneously broken.

\subsubsection{Coulomb/confining phase}

Now we increase $m_{0}$ above the bound (126). The exponentially small $\sigma_{\text {vac }}$ solution no longer exists. The only solution is

$$
\langle\sigma\rangle_{\mathrm{vac}}=0
$$

In addition, Eq. (127) implies

$$
|n|=0, \quad i D=\Lambda^{2}-m^{2} .
$$

This solution describes a single $Z_{N}$ symmetric vacuum. All other vacua are lifted and become quasivacua (metastable at large $N$ ). This phase is quite similar to the Coulomb/confining phase of nonsupersymmetric $\operatorname{CP}(N-1)$ model without twisted masses [35]. The presence of small splittings between 


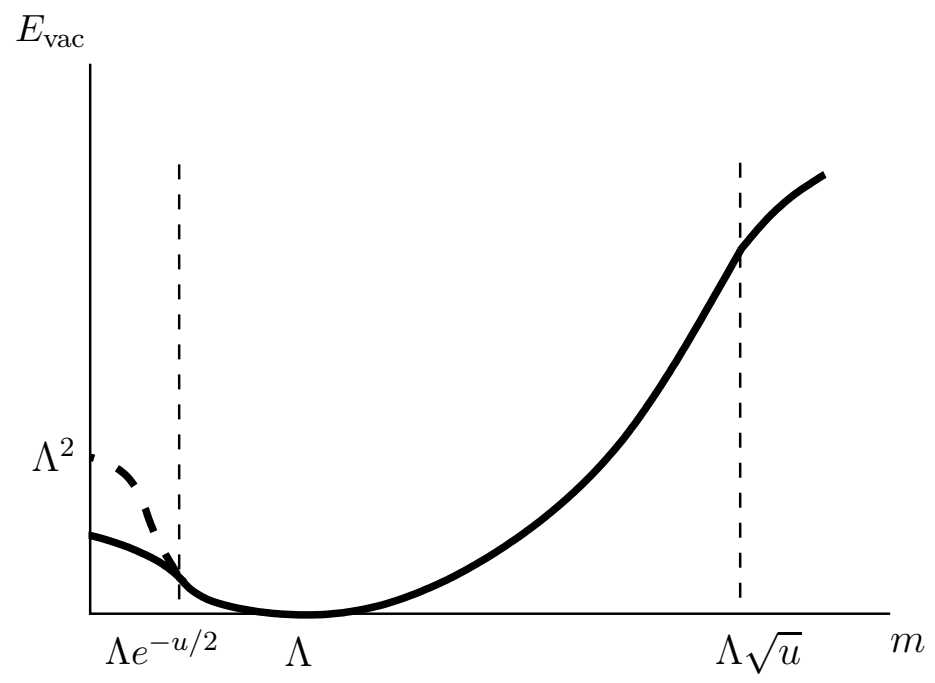

Figure 10: Vacuum energy density vs. $m_{0}$. The dashed line shows the behavior of the energy density (131) extrapolated into the strong coupling region.

quasivacua produces a linear rising confining potential between kinks that interpolate between, say, the true vacuum and the lowest quasivacuum [10], see also the review [12]. Alternatively, this is a Coulomb interaction between charged particles due to a massless photon that results in confinement.

There is a phase transition (most likely of the second order) that separates these phases. As a rule, one does not have phase transitions in supersymmetric theories. However, in the model at hand supersymmetry is broken (in fact, it is broken already at the classical level [18]); therefore, the emergence of a phase transition is not too surprising.

One can calculate the vacuum energy explicitly to see the degree of supersymmetry breaking. Substituting (129) and (130) in the effective potential one gets

$$
E_{\mathrm{vac}}^{\text {Coulomb }}=\frac{N}{4 \pi}\left[\Lambda^{2}-m_{0}^{2}+m_{0}^{2} \ln \frac{m_{0}^{2}}{\Lambda^{2}}\right] .
$$

see Fig. 10. At $m_{0}=\Lambda$ the vacuum energy vanishes in the large- $N$ limit implying a supersymmetry restoration. Most likely, this vanishing will be lifted by $1 / N$ corrections, so that supersymmetry is always spontaneously broken. 


\subsubsection{Higgs phase}

The Higgs (weakly coupled) phase takes place in the model under consideration at large $m_{0}$,

$$
m_{0}>\sqrt{u} \Lambda, \quad \text { if } u \gg 1 .
$$

In this phase $|n|$ develops a VEV, which is a clear-cut signal of the $Z_{N}$ symmetry breaking. Thus, we conclude that

$$
|n|_{\text {vac }}^{2}=\frac{N}{4 \pi} \ln \frac{\sqrt{2} \sigma m_{0}}{\Lambda^{2}} \sim \frac{N}{4 \pi} \ln \frac{m_{0}^{2}}{u \Lambda^{2}}
$$

in each of the $N$ vacua in the Higgs phase. were

$$
\sqrt{2}|\sigma|_{\mathrm{vac}}=\left(\frac{8 \pi}{N}\right) \frac{m_{0}}{u},
$$

We have $N$ degenerate vacua again, as in the strongly coupled phase. In each of them $|\sigma|$ is small $\left(\sim m_{0} / u\right)$ but nonvanishing. The $Z_{N}$ chiral symmetry is broken. Clearly, the Higgs phase is separated form the Coulomb/confining phase (where $Z_{N}$ is unbroken) by a phase transition.

\subsubsection{Goldstino}

In this section we limit ourselves to the large- $N$ solution of the massless heterotic model (113), derived from the bulk theory in 26. Due to the spontaneous supersymmetry breaking we have a massless Goldstino fermion in the world-sheet theory. To check this explicitly one can analyze the oneloop effective Lagrangian calculated in [26]. The appropriate fermionic part of the effective Lagrangian is

$$
\begin{aligned}
\mathcal{L}_{\text {eff }}^{\text {ferm }} & =\frac{1}{e_{\lambda}^{2}} \bar{\lambda}_{R} i \partial_{L} \lambda_{R}+\frac{1}{e_{\lambda}^{2}} \bar{\lambda}_{L} i \partial_{R} \lambda_{L}+\frac{1}{2} \bar{\zeta}_{R} i \partial_{L} \zeta_{R} \\
& +\left[i \sqrt{2} \Gamma \bar{\sigma} \bar{\lambda}_{L} \lambda_{R}+\sqrt{2} i \omega \bar{\lambda}_{L} \zeta_{R}+\text { H.c. }\right],
\end{aligned}
$$

where the one-loop couplings $e_{\lambda}$ and $\Gamma$ were calculated in [26].

First, we diagonalize the mass matrix for the $\zeta_{R}, \lambda_{R}$ and $\lambda_{L}$ fermions. Equating the determinant of this matrix to zero produces the following equation for the mass eigenvalues $m$ :

$$
m^{3}-m\left(2|\sigma|^{2} \Gamma^{2} e_{\lambda}^{4}+4 \omega^{2} e_{\lambda}^{2}\right)=0 .
$$


For any $\omega$ we have a vanishing eigenvalue corresponding to a massless Goldstino. Clearly, at small $\omega$ this fermion coincides with $\zeta_{R}$ (with an $O(\omega)$ admixture from the $\lambda$ fermions).

At large $u$

$$
e_{\lambda} \sim \Lambda \text { and } \Gamma \sim u / \Lambda^{2}
$$

while $\sigma$ is given by (128). Thus, the last term in the second line in (135) dominates, giving masses to $\zeta_{R}$, and $\lambda_{L}$. The role of Goldstino is assumed by the $\lambda_{R}$ fermion field.

\subsection{Large $N$ in nonminimal heterotic weighted $\mathrm{CP}(N, M)$ model}

The unperturbed $\mathcal{N}=(2,2)$ model was discussed in Sec. 8. It is obtained on the world sheet of semilocal strings supported in the bulk $\mathcal{N}=2$ theories if $N_{f}>N$ [54, 55, 56, 57]. In this case there are two distinct types of the moduli fields, $\rho$ and $n$, (scale and orientation moduli, respectively), and we arrive at the so-called $z n$ model on the world sheet. Hanany and Tong suggested [54] the weighted $\mathrm{CP}(N, M)$ for the same purpose. Later it was shown that these two models lead to identical predictions in the large- $N$ limit.

If one introduces a $\mu \operatorname{Tr} \mathcal{A}^{2}$ deformation in the bulk theory breaking $\mathcal{N}=2$ down to $\mathcal{N}=1$ one arrives at a heterotically deformed model on the world sheet. As far as we know, no explicit derivation of the deformation term in two dimensions starting from the deformed bulk theory has ever been carried out. A conjecture that this deformation term is identical to that emerging in the $N_{f}=N$ case was formulated in [60]. Then the two-dimensional model obtained in this way was further generalized to include twisted masses of two types, corresponding to two types of the moduli fields, namely, the scale and orientational moduli. To reduce the number of adjustable parameters, it was assumed that the first set of the twisted masses is $Z_{M}$ symmetric, while the second is $Z_{N}$ symmetric (cf. (63) ). As a result, there are two mass parameters $m_{0}$ and $\mu_{0}$ plus two dimensionless parameters

$$
\alpha=\frac{M}{N} \text { and } u \text {. }
$$

The limit $N \rightarrow \infty$ was assumed. The large- $N$ analysis of the vacuum structure and the spectrum of the model is very similar to that discussed in Sec. 9.4. Under these conditions the model was solved [60] and a rich struc-

ture discovered on the phase diagrams, including two distinct Higgs phases 
and two distinct Coulomb phases and various patterns of the $Z_{N, M}$ breaking. An interesting phenomenon was observed on a two-dimensional subspace of mass parameter space on which a discrete $Z_{N-M}$ symmetry is preserved. As was expected, supersymmetry is spontaneously broken for generic values of adjustable parameters. However, on a special curve in the parameter space we have the same phenomenon as at $m_{0}=\Lambda$ in Fig. 10, Supersymmetry seems to be restored at $N \rightarrow \infty$. A new branch opens up for special values of $m_{0}$ and $\mu_{0}$. In much the same way as in Sec. 9.4.2 one can expect that the vacuum energy on this curve will be lifted in a subleading order in $1 / N$.

\subsection{Large $N$ in heterotic $\mathrm{O}(N)$ model}

To begin with, a few words about the minimal $(0,1) \mathrm{O}(N)$ model will be in order. Assuming $N \geq 4$ it is easy to obtain this model by truncating the standard $(1,1)$ model [37], for a review see [38]. To this end we introduce the $(0,1)$ superfield

$$
N^{a}=S^{a}(x)+\theta_{R} \psi_{L}^{a}(x), \quad a=1,2, \ldots, N,
$$

with the following Lagrangian [20] (plus the standard constraint)

$$
\mathcal{L}_{(0,1) \min }=\frac{1}{2 g^{2}} \int d \theta_{R}\left(D_{L} N^{a}\right)\left(i \partial_{R} N^{a}\right), \quad N^{a} N^{a}-1=0,
$$

where $\psi_{L}^{a}$ is a Weyl-Majorana field, $\partial_{R}=\partial_{t}-\partial_{z}$ as usual, and

$$
D_{L}=\frac{\partial}{\partial \theta_{R}}-i \theta_{R} \partial_{L}
$$

The constraint in (139) can be implemented by adding an appropriate Lagrange multiplier term

$$
\Delta \mathcal{L}_{(0,1) \min }=\int d \theta_{R} X\left(N^{a} N^{a}-1\right)
$$

where

$$
X=\frac{1}{2 g^{2}}\left(-\lambda_{R}+\theta_{R} D\right) \text {. }
$$

Note that, in contradistinction with the $\mathrm{CP}(N-1)$ case, the minimal $\mathrm{O}(N)$ model exists at all $N$. The large- $N$ solution of the model (139) is constructed in much the same way as that for nonsupersymmetric $\mathrm{O}(N)$ model 38 . 
Supersymmetry is spontaneously broken, the constraint $S^{a} S^{a}=1$ is lifted, all $S^{a}$ fields acquire a mass while the $\psi_{L}$ fields remain massless. The field $\chi_{R}$ acquires a kinetic term.

To construct a nonminimal heterotic model we will follow the same line of reasoning as in Sec. 9.2.1. In fact, in the geometric formulation one can use the Lagrangian (111) with the replacement of the Kähler metric of $\mathrm{CP}(N-1)$ by a real metric of the $N$-dimensional sphere, and assuming that the parameter $\theta$ in the definition of the superfields (105) and (112) is real.

A slightly different formulation is more convenient for the large- $N$ analysis, however. In addition to the $(0,1)$ superfield (138) let us introduce two right-handed "matter" superfields (both with one physical degree of freedom),

$$
\mathcal{B}=\zeta_{R}(x)+\theta_{R} F_{\zeta}(x), \quad B^{a}=\psi_{R}^{a}(x)+\theta_{R} F_{\psi}^{a}(x)
$$

The Lagrangian of the model can be written as

$$
\begin{aligned}
\mathcal{L}_{(0,1)} & =\int d \theta_{R}\left\{\frac{1}{2 g^{2}}\left[\left(D_{L} N^{a}\right)\left(i \partial_{R} N^{a}\right)+\left(D_{L} B^{a}\right) B^{a}\right]+\frac{1}{2}\left(D_{L} \mathcal{B}\right) \mathcal{B}\right. \\
& \left.-\frac{\kappa}{g^{2}}\left(D_{L} N^{a}\right) B^{a} \mathcal{B}-X\left(N^{a} N^{a}-1\right)-\tilde{X}\left(N^{a} B^{a}\right)\right\},
\end{aligned}
$$

where the last two terms implement the constraints $S^{a} S^{a}=1$ and $S^{a} \psi_{L, R}^{a}=$ 0 (plus the standard relation for $F_{\psi}$, see [38]) and $\tilde{X}$ is an auxiliary field analogous to (141), namely,

$$
\tilde{X}=\frac{1}{g^{2}}\left(\sigma+\theta_{R} \lambda_{L}\right)
$$

In components (after eliminating the auxiliary fields $F_{\zeta, \psi}$ and a rescaling needed to make kinetic terms canonical) the Lagrangian takes the form [64]

$$
\begin{aligned}
\mathcal{L}_{(0,1)} & =\frac{1}{2} \partial_{L} S^{a} \partial_{R} S^{a}+\frac{i}{2} \psi_{L}^{a} \partial_{R} \psi_{L}^{a}+\frac{i}{2} \psi_{R}^{a} \partial_{L} \psi_{R}^{a}+\frac{i}{2} \zeta_{R} \partial_{L} \zeta_{R} \\
& +\beta_{L} \psi_{R}^{a} S^{a}+\chi_{R} \psi_{L}^{a} S^{a}-\frac{1}{2}\left(D+\sigma^{2}\right) S^{a} S^{a}+\frac{1}{2} \frac{D}{g^{2}} \\
& +\sigma \psi_{L}^{a} \psi_{R}^{a}+\kappa\left(i \partial_{L} S^{a}\right) \psi_{R}^{a} \zeta_{R}+\frac{1}{2} \kappa^{2} \sigma^{2} .
\end{aligned}
$$


It is not difficult to calculate the effective potential as a function of $D$ and $\sigma$ 64],

$$
V_{\text {eff }}=\frac{N}{8 \pi}\left[D \log \frac{\Lambda^{2}}{D+\sigma^{2}}+\sigma^{2} \log \frac{\sigma^{2}}{\sigma^{2}+D}+D+u \sigma^{2}\right]
$$

where

$$
u=\frac{4 \pi \kappa^{2}}{g^{4} N} .
$$

Minimizing the potential with respect to $D$ and $\sigma$ one finds two distinct vacua of the theory

$$
\sigma_{0}= \pm \Lambda e^{-\frac{u}{2}}, \quad D=\Lambda^{2}-\sigma^{2}
$$

which present continuations of two distinct vacua inherent to the supersymmetric $(1,1)$ limit of the model. The ensuing vacuum energy is

$$
E_{\mathrm{vac}}=\frac{N}{8 \pi} \Lambda^{2}\left(1-e^{-u}\right)
$$

Any nonvanishing value of $u$ results in the spontaneous breaking of supersymmetry. The spectrum of the model and, in particular, the Goldstino composition can be readily found too.

\section{In the uncharted waters}

In Sec. 2 we outlined the simplest prototype bulk theory supporting nonAbelian strings which, in turn, give rise to the observed wealth of twodimensional sigma models in Secs. 4. 9. Extending the bulk theory one can expect do derive novel sigma models on the string world sheet. In this section we will briefly discuss an extended construction resulting in the $(0,2)$ two-dimensional model which has never been discussed previously. Moreover, its geometric formulation is not yet known.

For brevity of presentation we will stick to $N=2$ and $N_{f} \geq 2$, referring the reader to the original papers [65, 66] for the case of generic $N$. Unlike Sec. 2 we will switch off the Fayet-Iliopoulos $D$ term (i.e. $\xi=0$ in the last term in Eq. (3) ) but, instead, switch on the mass term for the adjoint fields $\mathcal{A}$,

$$
\mathcal{W}_{\mathrm{def}}=\mu \operatorname{Tr} \Phi^{2}, \quad \Phi \equiv \frac{1}{2} \mathcal{A}+T^{a} \mathcal{A}^{a}
$$


in addition to non-vanishing mass terms for the bulk (s)quark fields [65, 66]. The deformation (150) breaks bulk supersymmetry down to $\mathcal{N}=1$, generally speaking.

This leads to the following modification of the bulk potential (3). The last two $F$ terms in the second line in (3) responsible for the squark condensation are replaced by

$$
2 g_{2}^{2}\left|\tilde{q}_{A} T^{a} q^{A}+\frac{\mu}{\sqrt{2}} a^{a}\right|^{2}+\frac{g_{1}^{2}}{2}\left|\tilde{q}_{A} q^{A}+\frac{N}{\sqrt{2}} \mu a\right|^{2} .
$$

Since VEVs of the adjoint fields $a$ and $a^{a}$ are determined by squark masses (cf. (41)) this leads to the breaking of the color-flavor symmetry. The quark VEVs are no longer degenerate. Instead of (6) the quark VEVs take the form

$$
\begin{aligned}
\left\langle q^{k A}\right\rangle & =\left\langle\overline{\tilde{q}}^{k A}\right\rangle=\frac{1}{\sqrt{2}}\left(\begin{array}{ccccc}
\sqrt{\xi_{1}} & 0 & 0 & \ldots & 0 \\
0 & \sqrt{\xi_{2}} & 0 & \ldots & 0
\end{array}\right), \\
k & =1,2, \quad A=1, \ldots, N_{f} .
\end{aligned}
$$

The parameters $\xi_{1,2}$ in (152) in the quasiclassical approximation are

$$
\xi_{1,2} \approx 2 \mu m_{1,2}
$$

These parameters can be made large in the large- $m$ limit even if $\mu$ is small, to ensure that the bulk theory is at weak coupling.

The squark condensation leads to the string formation. If $m_{1} \neq m_{2}$ these strings have nondegenerate tensions. The $\mathrm{U}(2)$ gauge group is broken down to $\mathrm{U}(1) \times \mathrm{U}(1)$ by the quark mass difference. To the leading order in $\mu$ each $\mathrm{U}(1)$ gauge factor supports it own BPS string. The string tensions of two strings under consideration are 65]

$$
T_{1,2}=2 \pi\left|\xi_{1,2}\right|
$$

If $\left|m_{1}-m_{2}\right| \ll\left|m_{1,2}\right|$ these two strings can still be promoted to non-Abelian strings with a shallow potential in the world-sheet theory. As was mentioned, now $\mathcal{N}=(2,2)$ supersymmetry is broken down to $\mathcal{N}=(0,2)$ even to the leading order in $\mu$. For the single-trace deformation (150) the bosonic part of the world-sheet theory becomes [65, 66]

$$
\mathcal{L}=\mathcal{L}_{(2,2)}+V_{\text {def }}(\sigma)
$$


where $\mathcal{L}_{(2,2)}$ is the Lagrangian of the $\mathcal{N}=(2,2)$ supersymmetric model (99) while the deformation potential is

$$
V_{\text {def }}(\sigma)=4 \sqrt{2} \pi|\mu \sigma|
$$

The deformation (156) respects only $(0,2)$ superalgebra.

This potential is radically different from the $|\sigma|^{2}$ potential in the heterotic deformation (113). The latter potential arises on the non-Abelian string in the massless bulk theory with the Fayet-Iliopoulos $D$-term deformed by the superpotential (150).

The total scalar potential is given by the sum of the twisted mass potential in (99) and deformation (156). Its minima correspond to tensions of two elementary non-Abelian strings,

$$
V\left(\sigma_{1,2}\right)_{\text {def }}=T_{1,2} \text {. }
$$

To see that this is the case we note that at small $\mu$ the vacuum values of $\sigma$ are still determined by the squark masses $\sqrt{2} \sigma_{1,2} \approx m_{1,2}$ in the quasiclassical approximation. Then (157) follows 5 from (153).

If $m_{1} \neq m_{2}$ the minima are nondegenerate. Only the lowest-lying vacuum is stable. The stability of the lowest vacuum in two dimensions means that only the lightest non-Abelian string is stable, the other one is metastable. Moreover, since generically the string tensions do not vanish, $\mathcal{N}=(0,2)$ supersymmetry is broken spontaneously already at the classical level [65].

To conclude this section let us mention that at the generic quark masses the deformation (150) leads to the emergence of a whole set of isolated vacua in the bulk theory, the so-called $r$ vacua, $r \leq N$. In each $r$ vacuum $r$ quarks and $(N-r-1)$ monopoles condense. The vacuum in (152) correspond to the $r=N$ vacuum, with the maximal number of condensed quarks. The simplest example of $r<N$ vacuum, namely, an $r=N-1$ vacuum (with $r=N-1$ condensed squarks and no monopoles) was considered in [67. This vacuum also supports non-Abelian strings. However, in contradistinction with the $r=N$ vacuum, the two-dimensional theory on the string world-sheet receives in this case nonperturbative corrections from the bulk, through the bulk gaugino condensate. Nonperturbative bulk effects deforming the theory on the string world sheet were found in [67] by virtue of the method of resolvents suggested by Gaiotto, Gukov and Seiberg for surface defects 68.

5 This statement is valid beyond the quasiclassical approximation (to all orders in $\left.\Lambda / m_{1,2}\right)$. In this case the $\sigma \mathrm{VEVs}$ are determined [65] by the roots of the equation (88). 


\section{Conclusions}

Forty years ago A. Polyakov emphasized that asymptotically free two-dimensional sigma models could present the best laboratory for the four-dimensional Yang-Mills theories. This prophecy came true in various aspects - even more than it was anticipated. First and foremost, a remarkable 2D-4D correspondence was detected in supersymmetric theories (see [12, 67] and references therein): the BPS spectrum of the sigma models on the string world sheet proves to be in one-to-one correspondence with that in the bulk fourdimensional theory. Moreover, diverse two-dimensional sigma models per se exhibit nontrivial dynamical features which, quite unexpectedly, proved to be in close parallel with some features of four-dimensional Yang-Mills. Novel models continue to appear in the limelight. Today the task of their exploration is highly challenging. This path is fruitful.

\section{Acknowledgments}

This work is supported in part by DOE grant DE-FG02-94ER40823. The work of A.Y. was supported by FTPI, University of Minnesota, by RFBR Grant No. 13-02-00042a and by Russian State Grant for Scientific Schools RSGSS-657512010.2.

\section{Appendix: Various representations of the nonminimal heterotic model}

A nonmnimal heterotic deformation of the $\mathrm{CP}(N-1)$ model was suggested in [16] by adding a twisted superpotential $(0,2)$ term in the gauge representation (25) following from (24) in the limit $e^{2} \rightarrow \infty$. In the appropriate normalization in components the corresponding Lagrangian is

$$
\begin{aligned}
\mathcal{L} & =\bar{\zeta}_{R} i \partial_{L} \zeta_{R}+\left[2 i \omega \bar{\lambda}_{L} \zeta_{R}+\text { H.c. }\right] \\
& +\left|\mathcal{D}_{\mu} n^{l}\right|^{2}+2|\sigma|^{2}\left|n^{l}\right|^{2}+i D\left(\left|n^{l}\right|^{2}-2 \beta\right) \\
& +\bar{\xi}_{l R} i \mathcal{D}_{L} \xi_{R}^{l}+\bar{\xi}_{l L} i \mathcal{D}_{R} \xi_{L}^{l} \\
& +\left[i \sqrt{2} \sigma \bar{\xi}_{l R} \xi_{L}^{l}+i \sqrt{2} \bar{n}_{l}\left(\lambda_{R} \xi_{L}^{l}-\lambda_{L} \xi_{R}^{l}\right)+\text { H.c. }\right]+4|\omega|^{2}|\sigma|^{2}
\end{aligned}
$$


In this form it was used 6 in the large- $N$ solution of the model in [26]. The constraint on $\bar{n} \xi_{R}$ ensuing from (A.1) is

$$
\bar{n} \xi_{R}=\sqrt{2} \bar{\omega} \bar{\zeta}_{R}
$$

One can pass to the standard form of this constraint $\bar{n} \xi_{R}=0$ inherent to $(2,2)$ supersymmetry by shifting the $\bar{\xi}$ and $\xi$ fields,

$$
\xi=\xi^{\prime}+\frac{\bar{\omega}}{\sqrt{2} \beta} n \bar{\zeta}_{R}, \quad \bar{\xi}=\bar{\xi}^{\prime}+\frac{\omega}{\sqrt{2} \beta} \bar{n} \zeta_{R} .
$$

In terms of $\xi^{\prime}, \bar{\xi}^{\prime}$

$$
\bar{\xi}_{l R} i \mathcal{D}_{L} \xi_{R}^{l} \rightarrow\left(\bar{\xi}_{l R}\right)^{\prime} i \mathcal{D}_{L}\left(\xi_{R}^{l}\right)^{\prime}
$$

and instead of the first and second terms in the first line in (A.1) one obtains

$$
\begin{aligned}
& \bar{\zeta}_{R} i \partial_{L} \zeta_{R}+\left[2 i \omega \bar{\lambda}_{L} \zeta_{R}+\text { H.c. }\right] \\
& \rightarrow\left(1+\frac{|\omega|^{2}}{\beta}\right)\left(\bar{\zeta}_{R} i \partial_{L} \zeta_{R}\right)+\left[\frac{\bar{\omega}}{\sqrt{2} \beta}\left(\bar{\xi}_{l R}\right)^{\prime} \bar{\zeta}_{R} i \partial_{L} n^{l}+\text { H.c. }\right]
\end{aligned}
$$

Now we can omit primes, $\xi^{\prime} \rightarrow \xi$ in the transformed Lagrangian $\mathcal{L}$ replacing (A.1). The constraint (A.2) is traded for the trilinear term in the transformed Lagrangian,

$$
\begin{aligned}
\mathcal{L}_{\text {trilin }} & =\left(1+\frac{|\omega|^{2}}{\beta}\right)\left(\bar{\zeta}_{R} i \partial_{L} \zeta_{R}\right) \bar{\zeta}_{R} i \partial_{L} \zeta_{R}+\left[\frac{\bar{\omega}}{\sqrt{2} \beta}\left(\bar{\xi}_{l R}\right)^{\prime} \bar{\zeta}_{R} i \partial_{L} n^{l}+\text { H.c. }\right] \\
& +\left|\mathcal{D}_{\mu} n^{l}\right|^{2}+2|\sigma|^{2}\left|n^{l}\right|^{2}+i D\left(\left|n^{l}\right|^{2}-2 \beta\right) \\
& +\bar{\xi}_{l R} i \mathcal{D}_{L} \xi_{R}^{l}+\bar{\xi}_{l L} i \mathcal{D}_{R} \xi_{L}^{l} \\
& +\left[i \sqrt{2} \sigma \bar{\xi}_{l R} \xi_{L}^{l}+i \sqrt{2} \bar{n}_{l}\left(\lambda_{R} \xi_{L}^{l}-\lambda_{L} \xi_{R}^{l}\right)+\text { H.c. }\right]+4|\omega|^{2}|\sigma|^{2} .
\end{aligned}
$$

Equation (A.6), being rewritten in the geometric form, identically coincides with (109) provided that

$$
\kappa \beta=\frac{\omega}{\sqrt{2}}, \quad \mathcal{Z}=1+\frac{|\omega|^{2}}{\beta}, \quad Z=1 .
$$

\footnotetext{
${ }^{6}$ Note a different normalization of the $\zeta$ kinetic term in Eq. (2.13) in [26].
} 


\section{References}

[1] L.D. Landau and I. Ya. Pomeranchuk, On point-like interaction in Quantum Electrodynamics, Dokl. Akad. Nauk SSSR, 102, 489 (1955). [Reprinted in L.D. Landau's Collected Papers, Nauka, Moscow, 1969), Vol. 2, p. 247].

[2] I. Ya. Pomeranchuk, Doklady Akad. Nauk USSR, 103, 1005 (1955); I. Ya. Pomeranchuk, V. V. Sudakov and K. A. Ter-Martirosyan, Phys. Rev. 103, 784 (1956); I. Ya. Pomeranchuk, Doklady Akad. Nauk USSR, 104, 51 (1955); Doklady Akad. Nauk USSR, 105, 461 (1955); Nuovo Cim., 3, 1186 1956.

[3] D. J. Gross and F. Wilczek, Phys. Rev. Lett. 30, 1343 (1973); H. D. Politzer, Phys. Rev. Lett. 30, 1346 (1973).

[4] A.A. Anselm, Sov. Phys. JETP 9 (36), 608 (1959) [reprinted in M. Shifman (Ed.) Under the Spell of Landau, (World Scientific, Singapore, 2013), p. 526.

[5] N. Seiberg and E. Witten, Nucl. Phys. B426, 19 (1994), (E) B430, 485 (1994) hep-th/9407087]; Nucl. Phys. B431, 484 (1994) hep-th/9408099].

[6] Y. Nambu, Phys. Rev. D 10, 4262 (1974);

G. 't Hooft, Gauge theories with unified weak, electromagnetic and strong interactions, in Proc. of the E.P.S. Int. Conf. on High Energy Physics, Palermo, 23-28 June, 1975 ed. A. Zichichi (Editrice Compositori, Bologna, 1976); S. Mandelstam, Phys. Rept. 23, 245 (1976).

[7] M. R. Douglas and S. H. Shenker, Nucl. Phys. B 447, 271 (1995) [hep-th/9503163]; A. Hanany, M. J. Strassler and A. Zaffaroni, Nucl. Phys. B 513, 87 (1998) hep-th/9707244.

[8] A. Abrikosov, Sov. Phys. JETP 321442 (1957) [Reprinted in Solitons and Particles, Eds. C. Rebbi and G. Soliani (World Scientific, Singapore, 1984), p. 356];

H. Nielsen and P. Olesen, Nucl. Phys. B61 45 (1973) [Reprinted in Solitons and Particles, Eds. C. Rebbi and G. Soliani (World Scientific, Singapore, 1984), p. 365].

[9] A. Hanany and D. Tong, JHEP 0307, 037 (2003) hep-th/0306150; R. Auzzi, S. Bolognesi, J. Evslin, K. Konishi and A. Yung, Nucl. Phys. B 673, 187 (2003) [hep-th/0307287]; M. Shifman, A. Yung, Phys. Rev. D70, 045004 (2004). hep-th/0403149; A. Hanany and D. Tong, JHEP 0404, 066 (2004) hep-th/0403158. 
[10] A. Gorsky, M. Shifman and A. Yung, Phys. Rev. D 71, 045010 (2005) hep-th/0412082.

[11] M. Shifman and A. Yung, Phys. Rev. Lett. 110, 201602 (2013) arXiv:1303.7010 [hep-th]]; S. Monin, M. Shifman and A. Yung, Phys. Rev. D 88, 025011 (2013) arXiv:1305.7292 [hep-th]].

[12] M. Shifman and A. Yung, Supersymmetric Solitons (Cambridge University Press, 2009).

[13] P. Fayet and J. Iliopoulos, Phys. Lett. B 51, 461 (1974).

[14] M. Shifman, Advanced Topics in Quantum Field Theory, (Cambridge University Press, 2012).

[15] E. B. Bogomol'nyi, Sov. J. Nucl. Phys. 24, 449 (1976), reprinted in Solitons and Particles, eds. C. Rebbi and G. Soliani (World Scientific, Singapore, 1984) p. 389. M. K. Prasad and C. M. Sommerfield, Phys. Rev. Lett. 35, 760 (1975), reprinted in Solitons and Particles, Eds. C. Rebbi and G. Soliani (World Scientific, Singapore, 1984) p. 530.

[16] M. Edalati and D. Tong, JHEP 0705, 005 (2007) hep-th/0703045.

[17] M. Shifman and A. Yung, Phys. Rev. D 77, 125016 (2008) [Erratum-ibid. D 79, 049901 (2009)] [arXiv:0803.0158 [hep-th]].

[18] P. A. Bolokhov, M. Shifman and A. Yung, Phys. Rev. D 79, 085015 (2009) arXiv:0901.4603 [hep-th]]; Phys. Rev. D 81, 065025 (2010) arXiv:0907.2715 [hep-th]].

[19] A. M. Perelomov, Phys. Rept. 146, 135 (1987); Phys. Rept. 174, 229 (1989).

[20] P. West, Introduction to Supersymmetry and Supergravity, Second Edition, (World Scientific, Singapore, 1990), Chapter 23.

[21] E. Witten, Adv. Theor. Math. Phys. 11 (2007) hep-th/0504078.

[22] M. -C. Tan and J. Yagi, Lett. Math. Phys. 84, 257 (2008) arXiv:0801.4782 [hep-th], arXiv:0805.1410 [hep-th]].

[23] J. Yagi, Adv. Theor. Math. Phys. 16, 1 (2012) arXiv:1001.0118 [hep-th]].

[24] B. Jia, E. Sharpe and R. Wu, Notes on non-Abelian (0,2) theories and dualities, arXiv:1401.1511 [hep-th]. 
[25] T. T. Dumitrescu and N. Seiberg, JHEP 1107, 095 (2011) arXiv:1106.0031 [hep-th]].

[26] M. Shifman and A. Yung, Phys. Rev. D 77, 125017 (2008) [Erratum-ibid. D 81, 089906 (2010)] arXiv:0803.0698 [hep-th]].

[27] P. A. Bolokhov, M. Shifman and A. Yung, Phys. Rev. D 82, 025011 (2010) arXiv:1001.1757 [hep-th]].

[28] X. Cui and M. Shifman, Phys. Rev. D 82, 105022 (2010) arXiv:1009.4421 [hep-th]].

[29] X. Cui and M. Shifman, Phys. Rev. D 84, 105016 (2011) arXiv:1105.5107 [hep-th]].

[30] X. Cui and M. Shifman, Phys. Rev. D 85, 045004 (2012) arXiv:1111.6350 [hep-th]].

[31] J. Chen, X. Cui, M. Shifman, and A. Vainshtein, arXiv:1404.4689.

[32] J. Wess and J. Bagger, Supersymmetry and Supergravity, Second Edition, Princeton University Press, 1992.

[33] A. M. Polyakov, Phys. Lett. B 59, 79 (1975).

[34] D. Friedan, Phys. Rev. Lett. 45, 1057 (1980); L. Alvarez-Gaumé, D. Z. Freedman and S. Mukhi, Annals Phys. 134, 85 (1981).

[35] E. Witten, Nucl. Phys. B 149, 285 (1979).

[36] E. Witten, Nucl. Phys. B 403, 159 (1993) hep-th/9301042].

[37] E. Witten, Phys. Rev. D 16, 2991 (1977); P. Di Vecchia and S. Ferrara, Nucl. Phys. B 130, 93 (1977).

[38] V. A. Novikov, M. A. Shifman, A. I. Vainshtein and V. I. Zakharov, Phys. Rept. 116, 103 (1984).

[39] B. Zumino, Phys. Lett. B 87, 203 (1979).

[40] G. W. Moore and P. C. Nelson, Commun. Math. Phys. 100, 83 (1985); A. Manohar, G. W. Moore and P. C. Nelson, Phys. Lett. B 152, 68 (1985). 
[41] L. Alvarez-Gaumé and D. Z. Freedman, Commun. Math. Phys. 91, 87 (1983); S. J. Gates, Nucl. Phys. B 238, 349 (1984); S. J. Gates, C. M. Hull and M. Roček, Nucl. Phys. B 248, 157 (1984).

[42] A. Gorsky, M. Shifman and A. Yung, Phys. Rev. D 73, 065011 (2006) hep-th/0512153.

[43] A. Gorsky, M. Shifman and A. Yung, Phys. Rev. D 73, 125011 (2006) hep-th/0601131.

[44] A. Hanany and K. Hori, Nucl. Phys. B 513, 119 (1998) arXiv:hep-th/9707192].

[45] G. Veneziano and S. Yankielowicz, Phys. Lett. B 113, 231 (1982).

[46] A. D'Adda, A. C. Davis, P. Di Vecchia and P. Salomonson, Nucl. Phys. B 222, 45 (1983).

[47] S. Cecotti and C. Vafa, Commun. Math. Phys. 158, 569 (1993) hep-th/9211097.

[48] N. Dorey, JHEP 9811, 005 (1998) hep-th/9806056.

[49] N. Dorey, T. J. Hollowood and D. Tong, JHEP 9905, 006 (1999) arXiv:hep-th/9902134.

[50] M. Kontsevich and Y. Soibelman, Stability structures, motivic DonaldsonThomas invariants and cluster transformations, arXiv:0811.2435 [math.AG]. Wall-crossing structures in Donaldson-Thomas invariants, integrable systems and Mirror Symmetry arXiv:1303.3253 [math.AG]; D. Gaiotto, G. W. Moore and A. Neitzke, Commun. Math. Phys. 299, 163 (2010) arXiv:0807.4723 [hep-th]], D. Gaiotto, G. W. Moore and A. Neitzke, Framed BPS States, arXiv:1006.0146 [hep-th].

[51] M. Shifman, A. Vainshtein and R. Zwicky, J. Phys. A 39, 13005 (2006) hep-th/0602004.

[52] P. A. Bolokhov, M. Shifman and A. Yung, Phys. Rev. D 84, 085004 (2011) arXiv:1104.5241 [hep-th]], Phys. Rev. D 85, 085028 (2012) arXiv:1202.5612 [hep-th]]; N. Dorey and K. Petunin, JHEP 1205, 085 (2012) arXiv:1202.5595 [hep-th]].

[53] K. Hori and C. Vafa, Mirror symmetry, arXiv:hep-th/0002222. 
[54] A. Hanany and D. Tong, JHEP 0307, 037 (2003) hep-th/0306150. JHEP 0404, 066 (2004) hep-th/0403158.

[55] M. Shifman and A. Yung, Phys. Rev. D 73, 125012 (2006) arXiv:hep-th/0603134.

[56] M. Eto, J. Evslin, K. Konishi, G. Marmorini, M. Nitta, K. Ohashi, W. Vinci, N. Yokoi, Phys. Rev. D 76, 105002 (2007) arXiv:0704.2218 [hep-th]].

[57] M. Shifman, W. Vinci and A. Yung, Phys. Rev. D 83, 125017 (2011) arXiv:1104.2077 [hep-th]].

[58] A. Achucarro and T. Vachaspati, Phys. Rept. 327, 347 (2000) hep-ph/9904229].

[59] P. Koroteev, M. Shifman, W. Vinci and A. Yung, Phys. Rev. D 84, 065018 (2011) arXiv:1107.3779 [hep-th]].

[60] P. Koroteev, A. Monin and W. Vinci, Phys. Rev. D 82, 125023 (2010) arXiv:1009.6207 [hep-th]].

[61] D. Tong, JHEP 0709, 022 (2007) hep-th/0703235 [hep-th].

[62] V. A. Novikov, M. A. Shifman, A. I. Vainshtein and V. I. Zakharov, Nucl. Phys. B 229, 381 (1983); Phys. Lett. B 166, 329 (1986)

[63] M. A. Shifman and A. I. Vainshtein, Nucl. Phys. B 277, 456 (1986).

[64] P. Koroteev and A. Monin, Phys. Rev. D 81, 105001 (2010) arXiv:1003.2645 [hep-th]].

[65] M. Shifman and A. Yung, Phys. Rev. D 82, 066006 (2010) arXiv:1005.5264 [hep-th]].

[66] P. A. Bolokhov, M. Shifman and A. Yung, Phys. Rev. D 88, 085016 (2013) arXiv:1308.4494 [hep-th]].

[67] M. Shifman and A. Yung, Quantum Deformation of the Effective Theory on Non-Abelian string and 2D-4D correspondence, arXiv:1401.1455 [hep-th].

[68] D. Gaiotto, S. Gukov and N. Seiberg, JHEP 1309, 070 (2013) arXiv:1307.2578. 